\title{
Star Counts in the Globular Cluster $\omega$ Centauri. I. Bright Stellar Components $^{1,2}$
}

\author{
V. Castellani ${ }^{3}$, A. Calamida ${ }^{3,4}$, G. Bono ${ }^{3,5}$, P. B. Stetson ${ }^{6,14,15}$, L. M. Freyhammer ${ }^{7}$, S. \\ Degl'Innocenti ${ }^{8,9}$ P. Prada Moroni ${ }^{8,9}$ M. Monelli ${ }^{10}$, C. E. Corsi ${ }^{3}$, M. Nonino ${ }^{11}$, R. \\ Buonanno $^{4}$, F. Caputo ${ }^{3}$, M. Castellani ${ }^{3}$, M. Dall'Ora ${ }^{12}$, M. Del Principe ${ }^{13}$, I. Ferraro ${ }^{3}$, G. \\ Iannicola $^{3}$, A. M. Piersimoni ${ }^{13}$, L. Pulone ${ }^{3}$, C. Vuerli ${ }^{11}$,
}

\begin{abstract}
${ }^{1}$ During the revision of this manuscript Vittorio Castellani passed away on May 19, 2006. His suggestions, ideas, and personality will be greatly missed.

${ }^{2}$ Based on data obtained from the ESO Science Archive Facility and the Hubble Space Telescope Archive Facility.

${ }^{3}$ INAF-Osservatorio Astronomico di Roma, Via Frascati 33, 00040, Monte Porzio Catone, Italy; bono@mporzio.astro.it, caputo@mporzio.astro.it,m.castellani@mporzio.astro.it,corsi@mporzio.astro.it, giacinto@mporzio.astro.it, ferraro@mporzio.astro.it, pulone@mporzio.astro.it

${ }^{4}$ Universita' di Roma Tor Vergata, Via della Ricerca Scientifica 1, 00133 Rome, Italy, buonanno@mporzio.astro.it, calamida@mporzio.astro.it

${ }^{5}$ European Southern Observatory, Karl-Schwarzschild-Str. 2, D-85748 Garching bei Munchen, Germany

${ }^{6}$ Dominion Astrophysical Observatory, Herzberg Institute of Astrophysics, National Research Council, 5071 West Saanich Road, Victoria, BC V9E 2E7, Canada; Peter.Stetson@nrc-cnrc.gc.ca
\end{abstract}

${ }^{7}$ Centre for Astrophysics, University of Central Lancashire, Preston PR1 2HE; lmfreyhammer@uclan.ac.uk

${ }^{8}$ Dipartimento di Fisica "E. Fermi", Univ. Pisa, Largo B. Pontecorvo 3, 56127 Pisa, Italy; prada@df.unipi.it, scilla@df.unipi.it

${ }^{9}$ INFN, Sez. Pisa, via Largo B. Pontecorvo 2, 56127 Pisa, Italy

${ }^{10}$ IAC - Instituto de Astrofisica de Canarias, Calle Via Lactea, E38200 La Laguna, Tenerife, Spain; monelli@iac.es

${ }^{11}$ INAF-Osservatorio Astronomico di Trieste, via G.B. Tiepolo 11, 40131 Trieste, Italy; nonino@ts.astro.it, vuerli@ts.astro.it

${ }^{12}$ INAF - Osservatorio Astronomico di Capodimonte, via Moiariello 16, 80131 Napoli; dallora@na.astro.it

${ }^{13}$ INAF-Osservatorio Astronomico di Collurania, via M. Maggini, 64100 Teramo, Italy; milena@te.astro.it, piersimoni@te.astro.it

${ }^{14}$ Guest User, Canadian Astronomy Data Centre, which is operated by the Herzberg Institute of Astrophysics, National Research Council of Canada.

${ }^{15}$ Guest Investigator of the UK Astronomy Data Centre. 
We present an extensive photometric investigation on Horizontal Branch (HB), Red Giant Branch (RGB), and Main-Sequence Turn-Off (MSTO) stars in the Galactic globular cluster $\omega$ Centauri $=$ NGC 5139. The central regions of the cluster were covered with a mosaic of $F 435 \mathrm{~W}, F 625 \mathrm{~W}$, and $F 658 \mathrm{~N}$-band data collected with the Advanced Camera for Surveys on board the Hubble Space Telescope. The outer reaches were covered with a large set of $U, B, V, I$-band data collected with the mosaic CCD camera available at the $2.2 \mathrm{~m} \mathrm{ESO/MPI}$ telescope. The final catalogue includes $\sim 1.7$ million stars. We identified more than 3,200 likely HB stars, the largest sample ever collected in a globular cluster, and more than 12,500 stars brighter than the subgiant branch and fainter than the RGB bumps $(15 \leq B \leq 18)$. We found that the HB morphology changes with the radial distance from the cluster center. The relative number of extreme HB stars decreases from $\sim 30 \%$ to $\sim 21 \%$ when moving from the center toward the outer reaches of the cluster, while the fraction of less hot HB stars increases from $\sim 62 \%$ to $\sim 72 \%$. Current findings seem to support the evidence brought forward by Castellani et al. (2006a) that the Blue Tails, if affected by cluster dynamics, should be considered more a transient phenomenon rather than an intrinsic feature of GCs.

We performed a detailed comparison between observed ratios of different stellar tracers and theoretical predictions based on evolutionary models constructed by adopting a canonical primordial helium $(Y=0.23)$ content and metal abundances $(Z=0.0002, Z=0.001)$ that bracket the observed spread in metallicity of $\omega$ Cen stars. We found that the empirical star counts of HB stars are on average larger $(30 \%-40 \%)$ than predicted by current evolutionary models. Moreover, the rate of $\mathrm{HB}$ stars is $\sim 43 \%$ larger than the MSTO rate. The discrepancy between the rate of $\mathrm{HB}$ stars when compared with the rate of RG and MSTO stars supports the evidence that we are dealing with a true excess of HB stars.

Recent empirical evidence suggests the occurrence of He-enhanced stellar populations in $\omega$ Cen. Therefore, we constructed different sets of evolutionary models using the same metal contents, but higher $(Y=0.33,0.42)$ helium abundances. The comparison between theory and observations was performed by assuming a mix of stellar populations made with $70 \%$ of canonical stars and $30 \%$ of Heenhanced stars. We found that the observed RG/MSTO ratio agrees with the predicted lifetimes of He-mixed stellar populations. However, the empirical counts of $\mathrm{HB}$ and RG stars are once again systematically larger than predicted for Hemixed stellar populations. The discrepancy between theory and observations decreases by a factor of two when compared with rates predicted by canonical He content models, but still 15\%-25\% (Y=0.42) and 15\%-20\% (Y=0.33) higher 
than observed. Furthermore, the ratio between HB and MSTO star counts are $24 \%(\mathrm{Y}=0.42)$ and $30 \%(\mathrm{Y}=0.33)$ larger than predicted lifetime ratios. This finding seems to be robust, since the HB/MSTO ratio presents a strong sensitivity to helium abundance and it is marginally affected by field star contamination.

Finally, we briefly outline the impact that the new findings might have on the final evolutionary fate of low-mass stars.

Subject headings: globular clusters: $\omega$ Centauri - stars: evolution - stars: horizontal branch - stars: red giant branch

\section{Introduction}

The occurrence in some Galactic Globular Clusters (GGCs) of B-type subdwarfs populating the hot end of the Horizontal Branch (HB) represents a puzzling and vigorously debated observational feature. Without doubt they are He burning stars which have lost a large fraction of their H-rich envelopes. However, the mechanism governing such a peculiar mass-loss event is still unknown.

The discovery of similar stellar structures in the GGC $\omega$ Cen dates back to the seminal papers by Da Costa \& Villumsen (1981) and Da Costa, Norris \& Villumsen (1986). The spatial distribution of such an Extreme Horizontal Branch (EHB) population was investigated for the first time by Bailyn et al. (1992), who concluded that EHB stars are more centrally concentrated than both normal HB stars and subgiant (SG) stars. This, if confirmed, would represent relevant information about the origin of such a peculiar population. In particular, this would support the hypothesis of a binary mechanism (e.g., either the merging of pairs of degenerate dwarfs, or moderately wide binaries experiencing mass transfer episodes just before the He flash) for their formation.

However, Whitney et al. (1994, 1998) made use of the orbiting Ultraviolet Imaging Telescope (UIT) facility to study $\omega$ Cen cluster stars in a far- $U V$ band $(\lambda \sim 1620 \AA)$, reaching the conclusion of a similar spatial distribution for both EHB and normal HB stars. Finally, empirical evidence for an increase in the fraction of EHB when moving outside the cluster core (43\% vs 30\%) has been brought into focus by D'Cruz et al. (2002) discussing optical $(F 555 W)$ and far-UV Hubble Space Telescope (HST) data collected with three Wide Field Planetary Camera 2 (WFPC2) pointings. However, the same authors warn against the possibility of a selection effect in the $F 555 W$-band due to the crowding of the central regions.

In spite of such long-standing interest, we still lack exhaustive information on the cluster 
HB population. As a matter of fact, in the current literature one finds either photometry of small cluster regions, or Color-Magnitude Diagrams (CMDs) which barely reach the faint magnitudes of the EHB population. As a result, in the available wide-field CMDs the EHB appears as a sparse and rare population (see, e.g., Pancino 2002; Rey et al. 2002), whereas D'Cruz et al. (2002) have already found that the EHB population should represent 30-40\% of the total number of cluster HB stars.

The recent detection (Anderson 2002; Bedin et al. 2004) of a well-defined double MS in $\omega$ Cen has stimulated a relevant theoretical and observational effort. This secondary population includes a fraction of the order of $30 \%$ of $\omega$ Cen stars and shows up as a fainter and/or bluer MS when compared with canonical, redder MS stars. To account for this peculiar evolutionary feature a substantial increase in the He abundance has been suggested $(\Delta Y \approx 0.15$, Norris 2004; Piotto et al. 2005; Sollima et al. 2007). A similar large variation in the helium content was also suggested by Lee et al. (2005) to explain the large number of extreme horizontal branch stars present in $\omega$ Cen and in NGC2808 (D'Antona et al. 2005). The strong He-enrichment scenario was also supported by Maeder \& Meynet (2006) who suggested that models of low-metallicity, massive stars with moderate initial rotation velocities can produce stellar winds with large He- and N-excesses. However, the self-enrichment scenario has been recently questioned by Bekki \& Norris (2006). By assuming that the helium enrichment is only caused by ejecta from stars of the red MS they found that the suggested properties of the blue MS stars cannot be the aftermath of a self-enrichment process from either massive AGB stars, or mass loss of very massive young stars, or from Type II supernovae. Although the working hypothesis of a mixed He-enriched population accounts for some morphological empirical evidence, we still lack a detailed comparison between observed star counts and predicted lifetimes based on both canonical and He-enhanced evolutionary tracks and horizontal branch models.

In this paper we investigate the radial distribution of $\mathrm{HB}, \mathrm{RG}$, and main-sequence stars. In order to investigate star counts over a substantial fraction of $\omega$ Cen we use two different data sets. Multiband $(F 435 W, F 625 W, F 658 N)$ data collected with Advanced Camera for Surveys (ACS) on board the HST are adopted for the central cluster regions, while multiband $(U, B, V, I)$ ground-based data collected with a mosaic CCD camera (WFI@2.2m ESO/MPI) have been adopted for the external cluster regions. In $\S 2$, we discuss the two different photometric data sets, together with the strategy we adopted to perform the photometry. The approach we employed to transform stellar positions from pixels to equatorial coordinates and the cross-identification of the optical catalogue with the near-infrared and the proper motion catalogues are presented in $\S 3$. Empirical estimates of star counts for $\mathrm{HB}$ and RG stars are presented in $\S 4$ together with the comparison with evolutionary prescriptions that cover a broad range of metal abundances and plausible changes in cluster ages. $\S 5$ deals with 
the comparison of observed $\mathrm{HB}, \mathrm{RG}$, and MS star counts with their evolutionary lifetimes. The impact that a significant increase in the helium content has on predicted lifetimes of quoted evolutionary phases is discussed in detail in $\S 6$. A brief summary of the results and a few viable developments to further constrain the evolutionary properties of stellar populations in $\omega$ Cen are outlined in $\S 7$.

\section{Observations and data reduction}

Photometric data discussed in this investigation belong to two different sets from both space (HST) and ground based telescopes. Multiband $(U, B, V, I)$ data have been collected with the mosaic $(2 \times 4)$ CCD camera Wide Field Imager (WFI, Baade et al. 1999) available at the $2.2 \mathrm{~m}$ ESO/MPI telescope (ESO, La Silla). These data have been retrieved from the ESO science archive and include $8 \mathrm{U}, 39 \mathrm{~B}, 51 \mathrm{~V}, 26 \mathrm{I}$ pointings. The data include both shallow and relatively deep images, with exposure times ranging from 1 to 300 seconds for the $B, V$, and $I$ bands, and from 300 to 2,400 seconds for the $U$ band. The total exposure time per band for the different pointings is $7500 \mathrm{~s}(U), 3431 \mathrm{~s}(B), 3969 \mathrm{~s}(V)$, and $2156 \mathrm{~s}$ $(I)$. The data were collected in several observing runs ranging from 1999 to 2003. During this period two filters were changed: data secured before 2002 were collected with the ESO filters $U / 38_{E S O 841}$ and $B / 99_{E S O 842}$, while later ones with the ESO filters $U / 50_{E S O 877}$ and $B / 123_{E S O 878}$. These data were obtained in good seeing conditions, and indeed the mean seeing ranges from $\sim 0^{\prime \prime}$.60 for the $I$-band to $\sim 1^{\prime \prime}$. 1 for the $U$-band data. Table 1 gives, from left to right, the frame identification, the coordinates, the modified Julian date, the exposure time, the filter, and the seeing for each WFI pointing. The field of view (FOV) covered by each pointing is $34 \times 33$ arcminutes squared, and the FOV covered by the entire dataset is $42 \times 48$ arcminutes across the center of the cluster. The large solid black polygon in Fig. 1 shows the cluster area covered by this data set. The raw frames were pre-reduced by using standard IRAF procedures.

Multiband $(F 435 W, F 625 W, F 658 N)$ photometric data were collected with the ACS on board HST, and retrieved from the HST archive. These data include nine pointings across the center of the cluster (see blue solid polygons in Fig. 1). The $3 \times 3$ mosaic covers a field of view of $\approx 10^{\prime} \times 9.5^{\prime}$. Four images per field were acquired in three different bands. Data in the $F 435 \mathrm{~W}$ and in the $F 625 \mathrm{~W}$ band were secured with one shallow (8s) and three deep (340s each) exposures, while the exposure time for the four $H_{\alpha}(F 658 N)$ images was $440 \mathrm{~s}$ each. The raw frames were pre-reduced by using the standard HST pipeline. By assuming a King (1962) profile and the structural parameters (core radius, $r_{c}=2.6$ arcmin; tidal radius, 
$r_{t}=45$ arcmin) for $\omega$ Cen 11 given by Trager, King, \& Djorgovski (1995), we estimate that the current photometry accounts for approximately the $99 \%$ of the light of the cluster.

To improve the photometric accuracy, carefully chosen selection criteria were applied to pinpoint a large number $(\approx 200)$ of isolated point-spread function stars across the individual chips/frames, and several different reduction strategies were used to perform the photometry (DAOPHOT/ALLSTAR). The observed ground-based $(124 \times 8)$ and space $(108)$ photometric catalogues were rescaled to a common geometrical system with DAOMATCH/DAOMASTER. The entire mosaic of HST data was simultaneously reduced with DAOPHOTII/ALLFRAME and the final catalogue includes $\sim 1.32 \times 10^{6}$ stars with at least one measurement in two different photometric bands. The photometry was kept in the Vega system following the prescriptions suggested by Sirianni et al. (2006, and references therein). The main difference with the investigations by Freyhammer et al. (2005) and Monelli et al. (2005), which are based on the same data, is that they performed the photometry on individual pointings and adopted slightly different zero-points in the absolute calibration.

The ground-based data were divided into shallow (89) and deep (35) pointings and each set was simultaneously reduced with DAOPHOTII/ALLFRAME. The final catalogue includes $\sim 6.4 \times 10^{5}$ stars with at least one measurement in two different photometric bands. The relative and absolute photometric calibration of ground-based instrumental magnitudes was performed following the strategy suggested by Monelli et al. (2003) and Corsi et al. (2003). To accomplish this non-trivial objective we used a large set $(\sim 30,000$ stars $)$ of new multiband $(U, B, V, I)$ local photometric standard stars (Stetson 20002; Stetson et al. 2006, in preparation). The sky area covered by the standard stars is approximately of $33 \times 39$ arcmin squared and includes a substantial fraction of the current scientific data. The comparison between the current calibrated data and the local standard stars observed by Walker (1994) indicates that the agreement in the absolute zero-points is of the order of $0.03 \mathrm{mag}(B-\mathrm{band})$ or better $(V, I$-band).

The entire photometric catalogue includes $\sim 1.7 \times 10^{6}$ stars and it is, to our knowledge, the largest multiband data set ever collected for a Galactic Globular Cluster (GGC). Data reduction required several months of $\mathrm{CPU}$ time on a dedicated 64-bit server. Fig. 2 shows a color image of the central cluster regions with almost the same spatial resolution as the original ACS data. The color of individual stars is based on $U, B, V, I$-band data collected

\footnotetext{
${ }^{1}$ No general consensus has been reached yet on the structural parameters of $\omega$ Cen , the GGC catalogue by Harris (1996) gives $r_{c}=1.4$ and $r_{t}=57$ arcmin, while Meylan et al. (1995) give $r_{c}=3$ and $r_{t}=51$ arcmin.

${ }^{2}$ See also http://cadcwww.hia.nrc.ca/cadcbin/wdb/astrocat/stetson/query/
} 
with the WFI. Note that the crowding of the innermost regions is a major problem also for a globular cluster with a modest central density $\left(\log \rho=3.12 L_{\odot} p c^{-3}\right.$, Harris 1996).

In order to provide a composite CMD including both ground-based and space data, we transformed the $F 435 W$ magnitudes into the standard $B$-band. By selecting common stars with $14.5 \leq B \leq 22.5$ we found the following calibration $B=F 435 W+0.03( \pm 0.02)-$ $0.0015( \pm 0.001) F 435 W$. Furthermore, we transformed ground-based $V$ - and $I$-band data into $F 625 W$ magnitudes as $F 625 W=V \times 0.544+I \times 0.455$. The two sets of magnitudes agree quite well, and indeed we found that the difference is within an rms of $0.03 \mathrm{mag}$. For the stars with measurements both in the ACS and in the WFI dataset we adopted the former one. Figure 3 shows the CMD in $B, B-F 625 W$. Stars plotted in this figure have been selected according to photometric accuracy $\left(\sigma_{B}, \sigma_{V}, \sigma_{F 625 W}, \sigma_{I}<0.03\right)$ and the "separation index" (sep > 3) 3. A good fraction of the bright Red Giant Branch (RGB, $B \sim 15.5, B-F 625 W \geq 1.4$ ) stars mainly comes from the WFI photometry, while faint main sequence stars come from the ACS photometry. A glance at the data plotted in this figure shows that relatively fast evolutionary phases such as the Asymptotic Giant Branch (AGB, $B \sim 14.5, B-F 625 W \sim 1.4$ ), the Blue Straggler sequence (BS, $16.5 \leq B \leq 18.5$, $0.3 \leq B-F 625 W \leq 0.8$ ), and the bright region of the White Dwarf (WD) cooling sequence $(B>21, B-F 625 W \sim-0.2)$ are all sampled well. However, we can not exclude that we are missing bright AGB stars $(B \leq 13, B-F 625 W \geq 2.4)$ and a fraction of RG stars located close to the tip of the RGB $(B \sim 13-13.5, B-F 625 W \sim 2.3-2.4)$.

The Horizontal Branch (HB), in particular, appears very well populated and shows a morphology quite similar to the blue HB of NGC2808 (Bedin et al 2000; Castellani et al. 2006a). Following the scheme adopted for NGC2808 stars, the blue HB tail can be divided into three different subgroups. Stars hotter than the RR Lyrae instability strip and brighter than the first gap $(B \leq 16.5$, EBT1), stars located between the first and the second gap (16.5 $\leq B \leq 17.8$, EBT2), and stars fainter than the second gap ( $B>17.8$, EBT3). The current limits (see solid lines in Fig. 7) have been fixed arbitrarily, since the first gap - unlike the case of NGC2808 - is not well defined in the optical bands.

\section{Cluster members: cleaning the herd}

In order to investigate the HB morphology as a function of the radial distance we devised the following approach:

\footnotetext{
3The 'separation index' quantifies the degree of crowding (Stetson et al. 2003). The current sep value corresponds to stars that have required a correction of less than $6 \%$ for light contributed by known neighbours.
} 
i) The optical catalogue was transformed into the equatorial system by using IRAF's IMMATCH package to establish the spatial transformation using a subsample of 704 stars in common with the van Leeuwen et al. (2000) catalogue of proper motions and membership probabilities in $\omega$ Cen. The latter catalogue covers a field measuring $47^{\prime}$ by $45^{\prime}$ on the sky, which was offset 9'3 west and 3.2 south of the cluster center (center ICRF position: $\left.\alpha=201.69065, \delta=-47^{\circ} .47855\right)$ to include a part of the halo. The mutual overlap with our field is approximately $366^{\prime} 9$ by $42^{\prime} .9$. The limiting magnitude of the 9847 stars in this

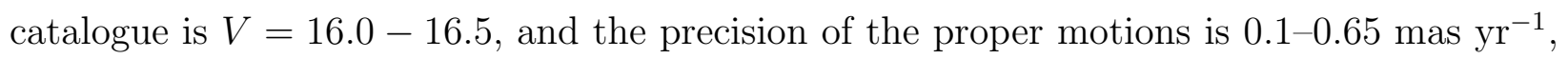
depending on stellar brightness. The r.m.s. of our transformation is 0.09 and 0.05 in $\alpha$ and $\delta$, respectively. We rejected matches for objects with separations exceeding 1".0 and identified $\sim 6,300$ stars in common. Among them, 280 stars (open circles in Fig. 5) have a probability smaller than $10 \%$ of being cluster members (van Leeuwen et al. 2000).

ii) Subsequently, the transformed equatorial coordinates of our optical catalogue were matched with those in the near-infrared (NIR) Two Micron All Sky Survey (2MASS, Cutri et al. 2003) catalogue 4 . We rejected matches for objects with separations exceeding $0^{\prime \prime} 4$ and identified $\sim 15,000$ stars in common. For these objects we have at least three optical and three NIR $(J, H, K)$ magnitudes. In order to split field and cluster stars we used the $U-J$ vs $B-H$ color-color plane (see top panel of Fig. 4) together with several optical-NIR CMDs, namely $B, U-J, V, B-H$, and $I, U-I$. We did not use the $K$-band photometry because it is less accurate in the faint magnitude limit $(B \geq 17)$. On the basis of these CMDs we provided a preliminary cut of the region of this plane where $\omega$ Cen RG stars are located by using the stars distributed in an annulus close to the cluster center $(2.5 \leq r \leq 10$ arcmin). We performed a linear fit of the $U-J$ color as a function of the $B-H$ color for the selected stars and then we estimated for each star in the sample the distance in color from the fitting line. We divided the sample in different $B-H$ color intervals and for each bin we fit the difference in $U-J$ color with a gaussian. The peaks and the sigmas of the different gaussians were adopted to improve the location of the fitting line and the selection of likely $\omega$ Cen stars. The procedure was repeated a few times by slightly changing the cuts in $B-H$ color. Data plotted in the middle and bottom panel of Fig. 4 show the last gaussian fits we performed, while the blue line in the top panel shows the final fitting line. The likely cluster RG stars (black dots) appear to be distributed along a well-defined sequence, while field objects form either two cooler plumes (red dots) or a hotter tail (green dots) for $1.5 \leq U-J \leq 4$. Likely cluster HB stars (blue dots) are located in the left bottom corner due to the strong sensitivity of these colors to the effective temperature. The $U$ magnitudes also show some dependence on surface gravity, since the Balmer convergence and continuum

\footnotetext{
${ }^{4}$ See also http://www.ipac.caltech.edu/2mass/releases/allsky/
} 
are included in that bandpass: high-luminosity stars are fainter in $U$ than low-luminosity stars of the same long-wavelength colors. A few dozen field objects present the same colors as HB stars, but they are significantly fainter/brighter than HB stars. Therefore we also selected HB stars on the basis of a magnitude criterion $(14.5 \leq B \leq 16.1)$. Note that to avoid systematic drifts in the selection of the different stellar samples we required that likely cluster RG and HB stars be located inside the expected magnitude and color intervals in the different optical-NIR CMDs.

The left panel of Fig. 5 shows the $B, U-H$ CMD of the cluster sample for candidate members, while the right one shows the CMD of the field objects $(\sim 1600)$. Although the criteria to select the different samples rely on photometric properties and partially on proper motions, data plotted in the left panel indicates that field objects have been properly subtracted. The AGB stars display a well-defined split from the RG stars, and the bright RG stars of the anomalous branch ( $\omega 3$, Rey et al. 2000; Pancino et al. 2000; Freyhammer et al. 2005) can be easily identified (see the arrows).

Furthermore, data plotted in the right panel show the typical color distribution of field stars (see, e.g., Gilmore et al. 1990). However, the small over-density of objects located at $B \sim 15$ and $U-H \sim 1.5$ suggests that the current selection includes a few cool cluster HB stars in the sample of field objects. As an independent test of the appropriateness of the current selection criteria, Fig. 6 shows the radial distribution of cluster stars (solid line) and field objects (dashed line). We accounted for the fact that $\omega$ Cen is ellipsoidal with a position angle of $\approx 100^{\circ}$ (van de Ven et al. 2006, and references therein) and the positions of individual objects were estimated as the distances projected along the major and minor axis, respectively. Data plotted in this figure show that the "field" objects show a mild increase when approaching the cluster center. However, this field sample is approximately $10 \%$ as large as the sample of cluster stars. Note that the decrease in the number of stars close to the cluster centre is due to the limited spatial resolution of the 2MASS catalogue. Moreover, galaxy counts based on multiband photometric data collected by the Sloan Digital Sky Survey (SDSS) suggest that the expected number of field galaxies per square degree with $B \leq 18$ is negligible $(\approx 30$, Yasuda et al. 2001) 5 . This evidence indicates that the conclusions of this investigation are at most marginally affected by the criteria adopted to distinguish field objects from cluster stars. For a more detailed discussion on the approach adopted to split field and cluster stars see Calamida et al. (2007, in preparation).

Once we removed the field objects from the optical-NIR catalogue we estimated the

\footnotetext{
${ }^{5}$ Note that to perform this estimate we accounted for the difference in magnitude between the JohnsonCousins system and the SDSS system (see, e.g., Jordi, Grebel, \& Ammon 2006).
} 
total flux in the $B$-band from the stars fainter than $B \sim 13$ and brighter than $B \sim 22$. Note that to estimate the total flux we did not apply selection criteria to the photometric catalogue, apart from the rejection of probable field objects. Moreover, in this magnitude range the completeness of the two photometric catalogues as a function of the radial distance is very similar. Then we divided the photometric catalogue into three concentric regions in such a way that each of them includes one third of the flux of the entire sample. We found that the three regions are given by $r \leq r_{\alpha}=204.3, r_{\alpha}<r \leq r_{\beta}=458.6$, and $r>r_{\beta}$ arcsec. Note that the current radial cuts marginally depend on the limits in magnitude adopted to estimate the fractional flux. An increase/decrease of one magnitude in the bright cut causes a change of \pm 3 arcsec in $r_{\alpha}$ and of \pm 11 arcsec in $r_{\beta}$. A similar change in the faint cut affects the values of $r_{\alpha}$ and $r_{\beta}$ by approximately one arcsec. For the stars located in the innermost regions we used only the HST photometry, while for the middle region we adopted either HST photometry $\left(r \leq r_{a c s} \sim 400\right.$ arcsec $)$ or ground-based photometry $\left(r_{a c s}<r \leq r_{\beta}\right)$. Fig. 7 shows, from left to right, the CMDs of space $(B, B-F 625 W)$ and ground-based $(B$, $B-V)$ data for annuli at increasing radial distance.

Data plotted in this figure show that the EBT2 region appears less populated than EBT1 and EBT3 regions in all three radial zones. Moreover, stars in the EBT3 region appear to decrease more rapidly toward the outskirts of the cluster when compared with stars in the EBT1 and in the EBT2 region. To express this circumstantial evidence on a more quantitative basis, Table 2 lists the star counts of the different sub-samples as a function of the radial distance. Note that the uncertainties given for the relative fractions of individual sub-samples only account for the statistical errors in the star counts. The stars plotted as black dots in Fig. 7 have been selected on the basis of the separation index, photometric errors, and sharpness. On the other hand, we did not apply any selection criteria to count the different stellar sub-samples (RG, red dots; HB, blue dots; $\omega_{3}$-branch, green dots; MS, cyan dots) apart from the upper and lower magnitude limits, and the rejection of field objects. We ended up with a sample of 3311 likely HB stars, which is by far the largest sample of HB stars measured in a GGC to date. On the whole, one finds that the relative number of HB stars (as normalized to the total $B$-band flux) steadily decreases when moving from the center to the outermost regions. This trend applies to individual sub-samples, therefore it can hardly be explained as an observational bias.

Moreover, the ratio between stars in the EBT3 and the total number of HB stars is roughly equal, within the errors, for $r \leq r_{\alpha}(N(E B T 3) / N(H B) \sim 0.28 \pm 0.02)$ and for $r_{\alpha}<r \leq r_{\beta}(N(E B T 3) / N(H B) \sim 0.32 \pm 0.02)$ and decreases to $\sim 0.21 \pm 0.02$ in the outermost region, while the fraction of stars in the EBT2 region is constant across the cluster $(N(E B T 2) / N(H B) \sim 0.08 \pm 0.01)$. On the other hand, the fraction of stars in the EBT1 region is $N(E B T 1) / N(H B) \sim 0.62 \pm 0.03$ for $r \leq r_{\beta}$ and increase to $N(E B T 1) / N(H B) \sim$ 
$0.72 \pm 0.04$ for $r>r_{\beta}$. The anonymous referee noted that the difference in the radial distribution between stars in the EBT1 and in the EBT3 region is further strengthened by the evidence that the ratio $N(E B T 3) / N(E B T 1)$ for $r \leq r_{\alpha}$ differs at $5.9 \sigma$ level $\left(T^{2}\right.$ statistics) with the same ratio for $r>r_{\beta}$. A glance at the $B$-band luminosity functions (LFs) of HB stars plotted in Fig. 8 as a function of the radial distance further confirms this radial trend. Note that the current space- and ground-based photometry is significantly deeper than the selected HB stars. Therefore current star counts should be minimally affected by completeness problems. Moreover, crowding effects would act in the opposite direction, since they should spuriously reduce the counts of EBT3 stars more in the region closer to the center than in the outskirts of the cluster.

However, to provide firm constraints on deceptive systematic errors that might affect the current HB star counts (crowding, saturated stars, limiting magnitudes) we performed the same selections by using different colors for both space $(B, B-F 658 N)$ and groundbased $(B, U-V)$ photometry. The new star counts are listed in Table 2 and they agree quite well - within the errors - with those quoted above. Moreover, HB stars plotted in Fig. 9 display the same trend concerning the radial gradient of the three EBT regions. Current findings support the results obtained by Bailyn et al. (1992) concerning the steady decrease in the number of EHB stars when moving toward the outer reaches of the cluster. They also support the results by D'Cruz et al. (2002) concerning the relative fraction of EBT3 stars $(\sim 27 \pm 1 \%$ vs $30 \%-40 \%)$.

\section{HB versus Red Giant Branch stars}

A relevant question concerning the occurrence of HB "blue tails" in globular clusters is to assess whether observed star counts along the HB agree with the normal evolutionary sequence in which HB stars are the predicted progeny of RG stars that have just experienced the He-core flash at the tip of the RGB. There are indeed evolutionary scenarios suggesting that either a fraction of HB stars approaches the blue tail along a different evolutionary path, or a fraction of RG stars misses the HB evolutionary phase altogether due to a virtually complete removal of the envelope.

It has already been suggested that the hot EBT3 group could be the aftermath of a quite different evolutionary path, e.g., the coalescence of two low-mass He-core WDs (Iben 1991, and references therein). In such a case the stars in the EBT3 group will appear as an addition to the normal and legitimate HB, and in turn, the total number of HB stars should exceed the number expected from the ongoing transition from RG into HB structures. 
However, according to an alternative suggestion advanced by Castellani \& Castellani (1993), but see also D'Cruz et al. (1996), Brown et al. (2001), and Cassisi et al. (2003), the stars in the EBT3 region might be the result of an episode of extreme mass loss - possibly caused by stellar encounters or compact binaries (Ivanova et al. 2006) - before the onset of the He-core flash. This phenomenon can drive the stellar mass very near to the lower mass limit for central He ignition. The RG stars above this limit undergo a late He-flash either during the contraction toward their He-WD structure or in the approach to their WD cooling sequence. These stellar structures have been christened in the literature as hot Heflashers (Castellani \& Castellani 1993; Sweigart 1997) and they have been photometrically (Momany et al. 2004) and spectroscopically (Moehler et al. 2002; Moehler \& Sweigart 2006, and references therein) identified in several GCs. However, it would not be a surprise if the same significant mass-loss mechanism also drives a fraction of the RG stars below the limit for central He ignition. This would imply that a fraction of RG stars will miss the He-core flash, thus directly evolving into cooling He-core WDs rather than spending time as HB stars (Castellani, Castellani, \& Prada Moroni 2006). The key consequence of such an evolutionary path is that one should observe a lower number of RG stars than predicted by evolutionary models. Moreover, according to the fraction of RG stars that after the heavy mass-loss episode are either above or below the limit for central He ignition one should observe either an increase in the number of extreme HB stars or in the number of stars in the bright region of the WD cooling sequence, relative to the total number of RG stars. It goes without saying that RG stars with stellar masses uniformly distributed across this limit should produce an increase in the number of both EHB stars and bright WDs (Castellani et al. 2006b).

One may explore such a problem by recalling that at advanced stages of evolution the number of stars observed in a given evolutionary phase is expected to be proportional to the time spent by stellar models in that given phase. We will check the number of HB stars measured in $\omega$ Cen by using the RG stars as a "reference clock". We counted RG stars in the magnitude range $15.0 \leq B \leq 17.8$. We selected this magnitude interval to secure a sizable sample of cluster stars in an evolutionary phase whose lifetime is minimally affected by both chemical discontinuity (RGB bump) and mass-loss efficiency (tip of the RGB). Star counts listed in Table 3 show that RG stars present the same radial trend as HB stars, i.e., their numbers steadily decrease (relative to the total $B$-band flux) when moving from the center to the outermost regions. Owing to the photometric precision and to the large sample of measured stars, data plotted in Figs. 7 and 9 show a well-defined $\omega 3$-branch in the three different annuli (green dots). Star counts listed in Table 3 also support the finding by Pancino et al. (2000) that this stellar population is approximately $3-4 \%$ of the entire population. There is weak evidence that this stellar population might increase when moving toward the outskirts. But on the whole, RG stars on the $\omega 3$-branch are less than $5 \%$ of the 
entire RG population and they will be neglected.

In order to provide a more quantitative analysis concerning star counts along the RGB, we counted RG stars in bins of one $B$-mag. This count was performed for eleven bins by adopting a shift of 0.1 in the magnitude range $16.6 \leq B \leq 17.6$ (see columns 3 to 5 in Table 4). Fig. 10 shows the RG star counts based on $B, B-F 625 W$ (ACS@HST) and $B, B-V$ (WFI@2.2m) CMDs as a function of the $B$-mag. Interestingly enough, RG stars do not show any significant radial trend for $r \leq r_{\beta}$, and indeed the star counts attain very similar values over the entire magnitude range. On the other hand, star counts in the external annulus are systematically smaller than the inner ones in all the bins. The same outcome applies if we use the RG stars selected in the CMDs based on $B, B-F 658 N$ (ACS@HST) and $B, U-V$ (WFI@2.2m) CMDs (dashed lines). The selection of the adopted radial annuli is based on the total $B$-band flux emitted by the different stellar populations in $\omega$ Cen. In order to validate the current approach, we compared the observed density profile of the entire stellar population with a standard isotropic single-mass King model with $r_{c}=155$ arcsec and $c=\log r_{t} / r_{c}=1.24$ (Trager et al. 1995; Ferraro et al. 2006) and we found a very good agreement. Therefore, the different populations should contribute to the total flux with the same number of stars. The difference we found in the outermost annulus might be caused either by the RG stars brighter than the HB or by the AGB stars. The star counts of these evolutionary phases will be discussed in a forthcoming paper.

In order to establish whether the number of RG stars is peculiar either in the two inner annuli or in the external one we need to compare empirical star counts with predicted evolutionary times. Inspection of recent evolutionary prescriptions (Pietrinferni et al. 2006), shows that in the lower portion of the RG branch (i.e., below the luminosity of the RGB bump and above the subgiant branch), this time is largely independent of cluster metallicity and of cluster age for ages ranging from 9 to 13 Gyr. The same outcome is also supported by empirical evidence, and indeed Stetson (1991) showed that cluster LFs, once shifted so that the Turn-Off magnitudes are coincident, marginally depend along the RGB on iron and $\alpha$-element abundances as well as on cluster age and initial mass function.

In order to provide more quantitative estimates of this dependence we constructed several evolutionary tracks by adopting different stellar masses and chemical compositions. Current evolutionary track 6 have been computed with an updated version of the FRANEC evolutionary code (see, e.g., Chieffi \& Straniero 1989) and by adopting the radiative opacities made available by the Livermore group (Iglesias \& Rogers 1996) and updated nuclear

${ }^{6}$ Several sets of evolutionary tracks and isochrones are available at the URL
http://astro.df.unipi.it/SAA/PEL/Z0.html


cross sections (Cariulo, Degl'Innocenti, \& Castellani 2004). Current evolutionary tracks account for element diffusion (Ciacio, Degl'Innocenti, \& Ricci 1997) with diffusion coefficients from Thoul, Bahcall \& Loeb (1994), and neglect mass loss during hydrogen- and helium-burning phases. Convective transport in the envelope regions is treated with the mixing-length formalism in which the parameter $\alpha$ defines the ratio between the mixing length and the local pressure scale height. We adopted $\alpha=2.0$, which reproduces - with the currently adopted color transformations - the observed RG branch colors of the Galactic globular clusters (Cariulo et al. 2004). Stellar structures during He-burning phases have been constructed according to the canonical prescriptions for "induced semiconvection" (see, e.g., Castellani et al. 1985).

To bracket the chemical compositions of the bulk of the stellar populations in $\omega$ Cen (Norris, Freeman \& Mighell 1996; Suntzeff \& Kraft 1996; Hilker et al. 2004; Sollima et al. 2005; Piotto et al. 2005; Sollima et al. 2006) we adopted $Z=0.0002$ and $Z=0.001$. Although precise empirical estimates of the metallicity distribution in $\omega$ Cen are available, we decided to use only two metal abundances, since the evolutionary lifetimes we plan to use display only a mild dependence on this intrinsic parameter. Moreover, to account for the age effect we adopted two stellar masses $M / M_{\odot}=0.8$ and 0.85 . These stellar masses imply a difference of $\approx 2$ Gyr in the turnoff age. Data plotted in Fig. 11 and evolutionary times listed in Table 5 show that an increase in metal abundance of a factor of five and a decrease in cluster age of $\approx 20 \%$ cause on average a change in the RG lifetime of the order of $10 \%$. This evidence strengthens the use of RG stars as a reference clock in stellar systems showing a spread in metal abundance and/or in stellar age such as $\omega$ Cen. Current evolutionary models for $\mathrm{Z} \leq 0.001$ have been constructed by adopting an original helium abundance of $Y \sim 0.23$. By taking into account a conservative estimate for the uncertainty of this input parameter $(0.23<Y<0.25$; Salaris et al. 2004, Spergel et al. 2006) we obtain a variation of the RGB lifetime, for a stellar mass of $\sim 0.8 M_{\odot}$, less than $10 \%$. Similarly, the error in the RGB lifetime due to the uncertainties in the input physics is of the order of $\sim 10 \%$ (Cassisi et al. 1998). On the other hand, the HB lifetime is marginally affected by a variation in the original helium abundance within plausible ranges (see, e.g., Bono et al. 1995). Cassisi et al. (2001) estimated a variation of $\sim 10 \%$ of the HB lifetime due to the uncertainty in the adopted input physics.

Theoretical predictions have been transformed into the observational plane by adopting the bolometric corrections and the color-temperature transformations provided by Bessell, Castelli \& Plez (1998). In particular, we adopted atmospheric models with an $\alpha$-enhanced composition $([\alpha / \mathrm{Fe}]=0.4)]$. These predictions are only available for surface gravities $\log g \leq$

${ }^{7}$ The entire set of atmosphere models is available at the URL 
5. In order to transform extreme HB models into the observational plane we linearly extrapolated by 0.5 dex the surface gravities out of the available range. The evolutionary times along the RGB have been estimated by adopting the same approach adopted for empirical star counts together with a distance modulus of $\mu_{0}=13.70 \pm 0.06 \pm 0.06$ (Del Principe et al. 2006), and a reddening of $E(B-V)=0.11 \pm 0.02$ (Kaluzny et al. 2002; Lub 2002; Calamida et al. 2005). By adopting the reddening law from Cardelli et al. (1989) we estimate that the apparent distance modulus in the $B$ band is $(m-M)_{B}=14.16 \pm 0.06 \pm 0.09$ mag. The systematic error accounts for the uncertainty both in the absolute zero point of the $B$-band and in the cluster reddening.

Fig. 12 shows the comparison between star counts and theoretical predictions at fixed stellar mass (top) and chemical composition (bottom). Evolutionary lifetimes have been normalized to observations in the magnitude bin $m_{B}=16.9-15.9\left(M_{B}=2.74-1.75\right)$. We selected this bin to avoid the possible interference of the RGB Bump. This intrinsic feature is indeed systematically brighter than our adopted magnitude limit over a broad range of stellar metallicities (see, e.g., Table 1 in Caputo \& Cassisi 2002). Theoretical predictions present a marginal dependence on metal abundance, and indeed predicted RG lifetimes for $\mathrm{Z}=0.0002$ (red lines) and for $\mathrm{Z}=0.001$ (blue line) attain quite similar values. Note that to avoid a complete overlap the lifetimes for $\mathrm{Z}=0.001$ have only been compared with the star counts of the outermost annulus. The same outcome applies to the dependence on the cluster age, and indeed a decrease of $2 \operatorname{Gyr}\left(M=0.80 M_{\odot}\right.$, red lines vs $M=0.85 M_{\odot}$, green line $)$ cause a marginal difference in the predicted $\mathrm{RG}$ lifetime. Once again to avoid the complete overlap the predictions for $M=0.85 M_{\odot}$ have only been compared with the star counts of the outermost annulus.

Data plotted in Fig. 12 indicate that theory and observations agree quite well in the two external annuli, while counts of the faintest giants attain slightly larger values in the inner annulus. This discrepancy can be caused neither by an increase in metal abundance of a factor of five (top, blue line) nor by a decrease in stellar age of the order of $20 \%$ (bottom, green line).

Simple physical arguments suggest that the ratio between the number of HB stars and the number of RG stars should scale according to the ratio of their relative evolutionary lifetimes. Data plotted in Fig. 11 indicate that HB lifetimes for extremely hot HB stars (EBT3, $T_{e} \sim 29,600 \mathrm{~K}, M / M_{\odot}=0.518$ ) are of the order of 94 Myr for $\mathrm{Z}=0.0002$ and of $101 \mathrm{Myr}$ for $\mathrm{Z}=0.001\left(T_{e} \sim 28,500 \mathrm{~K}, M / M_{\odot}=0.510\right)$. This evolutionary lifetime for hot HB stars $(\mathrm{EBT} 2)$ decreases to $84 \mathrm{Myr}\left(\mathrm{Z}=0.0002, T_{e} \sim 18,200 \mathrm{~K}, M / M_{\odot}=0.570\right)$ and to 
$90 \operatorname{Myr}\left(\mathrm{Z}=0.001, T_{e} \sim 18,200 \mathrm{~K}, M / M_{\odot}=0.551\right)$, while for less hot HB stars (EBT1) the evolutionary lifetime decreases to $76 \mathrm{Myr}\left(\mathrm{Z}=0.0002, T_{e} \sim 10,500 \mathrm{~K}, M / M_{\odot}=0.670\right)$ and to $82 \mathrm{Myr}\left(\mathrm{Z}=0.001, T_{e} \sim 10,000 \mathrm{~K}, M / M_{\odot}=0.620\right)$, respectively. Thus, "typical" average lifetimes are $\sim 98,87$, and $79 \mathrm{Myr}$ for EBT3, EBT2, and EBT1, respectively. Note that the three effective temperatures, and in turn the lifetimes, were selected to be representative of the three EBT regions (see Fig. 7) and of the peaks in the HB luminosity function (see Fig. $8)$.

In order to account for such an occurrence we estimated the arrival rate of stars onto the HB in the three EBT groups, namely $r(H B)=N(E B T 3) / 98+N(E B T 2) / 87+$ $N(E B T 1) / 79$. Note that to account for the possible spread in metal abundance the lifetime typical of the EBT3 group was estimated as the average $t_{E B T 3}$ lifetime for $\mathrm{Z}=0.0002$ and $\mathrm{Z}=0.001$ stellar structures. The same approach was adopted for the other HB groups and for the lifetime of RG structures. The use of $r(H B)$ represents the key advantage for overcoming the problem of the change in the predicted lifetime according to the HB morphology. Thus, the arrival rate of HB stars provides the opportunity to use, in the comparison between theory and observations, specific evolutionary time scales. The rates of HB stars in the three annuli are listed in column (6) of Table 2. As a whole, we found that the total rate of HB stars in $\omega$ Cen is $\sim 39$ stars per Myr. A similar rate was also estimated for RG stars by using the RG counts in the magnitude bins $16.60 \leq B \leq 17.60$ (see Table 4 ) and the evolutionary lifetimes listed in columns (3) and (4) of Table 5. Figure 13 shows the ratio between the $\mathrm{HB}$ and the $\mathrm{RG}$ rates. Interestingly enough, we found that the empirical HB star counts are on average between $30 \%$ and $40 \%$ more numerous than predicted by current evolutionary models. The mean in the three different radial annuli are $1.416 \pm 0.047$, $1.336 \pm 0.048$, and $1.339 \pm 0.030$ in the $B, B-F 625 W$ (ACS) and $B, B-V$ (WFI) CMDs, while they are $1.373 \pm 0.043,1.290 \pm 0.046$, and $1.385 \pm 0.027$ in the $B, B-F 658 N$ and $B, U-V$ CMDs. The current estimates, taken at face value, present the mild evidence that the ratio between $\mathrm{HB}$ and RG stars appears, on average in the different CMDs, larger in the innermost annulus ( $\sim 1 \sigma$ level). However, the current ratios do not allow us to determine whether such a discrepancy is caused by a decrease in the rate of relatively faint RG stars or by an increase in the rate of HB stars.

\section{HB and RGB versus Main Sequence stars}

The ratio between the rates of $\mathrm{HB}$ and RGB stars attains very plausible values as a function of the radial distance. However, to constrain on a quantitative basis whether the star counts of HB and RG stars present any peculiar radial trend we need to use MS stars 
as an independent clock. In particular, we selected MS stars just below the turnoff (TO) region in the magnitude bin $18.65 \leq B \leq 19.15 \mathrm{mag}$. The reason why we selected this region is twofold: i) empirical and theoretical evidence suggests that it marginally depends on the initial mass function (Zoccali \& Piotto 2000); ii) the magnitude range is quite similar to the magnitude of EHB stars, hence the two samples also present a very similar completeness; ii) stellar structures in this magnitude range present a small spread in mass, and indeed $\Delta M \leq 0.025 M_{\odot}$. Fig. 14 shows the ratio between RG stars and MS stars in the three different annuli. The different ratios attain quite similar values over the entire magnitude range, thus suggesting homologous radial distributions for RG and MS stars. Interestingly enough, the predicted ratios for $M=0.80 M_{\odot}$ and different metal abundances (red and blue lines) between MS (see open and filled circles in Fig. 11) and RG (see data listed in columns 3 and 4 of Table 5) lifetimes are in reasonable agreement with empirical ratios. Note that the theoretical ratios were not normalized to observed ratios and that no vertical shift was applied. The comparison between theory and observations indicates a mild excess in the number of observed RG stars. If we assume as a typical predicted ratio the mean of the two different metal abundances we found that the discrepancy between theory and observations on average ranges from $\sim 10 \%$ to $\sim 15 \%$ when moving from brighter to fainter RG stars. The same outcome applies if we use the predicted ratios based on younger stellar structures, i.e., lifetimes for $M=0.85 M_{\odot}$ (see data listed in columns 5 and 6 of Table 5 ).

The rates of MS stars in the three different annuli are -by using the star counts based on $B, B-F 625 W$ (ACS) and $B, B-V$ (WFI) CMDs and the mean of the lifetime for $\mathrm{Z}=0.0002$ $(2074 \mathrm{Myr})$ and for $\mathrm{Z}=0.001$ (2211 Myr) - the following: $19932 / 2093=9.52 \pm 0.95\left(r \leq r_{\alpha}\right)$, $19376 / 2093=9.26 \pm 0.93\left(r_{\alpha}<r \leq r_{\beta}\right)$, and $15223 / 2093=7.27 \pm 0.73\left(r>r_{\beta}\right)$. The errors account for uncertainties in both star counts (Poisson) and evolutionary lifetimes (10\%). The same outcome applies to the ratios given by star counts based on $B, B-F 658 N$ (ACS) and $B, U-V$ (WFI) CMDs, and indeed we find 19935/2093=9.52 $\pm 0.95\left(r \leq r_{\alpha}\right)$, $19815 / 2093=9.47 \pm 0.95\left(r_{\alpha}<r \leq r_{\beta}\right)$, and $14187 / 2093=6.78 \pm 0.68\left(r>r_{\beta}\right)$. Therefore, the total rate of MS stars in the selected box is slightly more than 25 stars per Myr. This means that on average the HB rate is approximately $43 \% 8$ larger than the MS rate. This discrepancy between theory and observations appears as robust empirical evidence, since it is marginally affected by the assumed metal abundance. Moreover, the ratio between HB and MS stars is also marginally affected by field star contamination. Hot HB stars are systematically bluer than field stars and the MS stars we selected cover a very narrow magnitude range. It is worth mentioning that the RR Lyrae stars in $\omega$ Cen $(>187$, Weldrake,

\footnotetext{
${ }^{8}$ In order to avoid subtle uncertainties in the estimate of the relative difference between the HB and the MS rate we decided to use $\xi=(r(H B)-r(M S)) /[(r(H B)+r(M S)) * 0.5]$.
} 
Sackett, \& Bridges 2006, and references therein) have not been identified yet in the current photometric catalogue. Some of these objects might have a limited coverage of the light curves, and therefore they have not been included in the sample of EBT1 stars. Therefore, the inclusion of these objects implies a mild increase in the observed HB/RG and HB/MS star counts ratios.

This finding and the excess showed by the ratio between the rate of $\mathrm{HB}$ and RG stars when compared to predicted ratios (see Fig. 13) is suggesting that we are faced with a true excess of HB stars. However, during the last few years several empirical and theoretical investigations suggested that a fraction of the order of $30 \%$ of the stars in $\omega$ Cen might present an increase in He content (Bedin et al. 2004; Piotto et al. 2005; Sollima et al. 2007, and references therein). This means that before we can reach a firm conclusion on the occurrence of a possible discrepancy between predicted and observed star counts we need to investigate the impact of the He content on MS, RG, and on HB evolutionary lifetimes.

\section{Dependence on He abundance}

In order to account for the dependence of evolutionary lifetimes on helium abundance, we constructed a new series of evolutionary models by assuming the same turnoff age $(t \sim 12$ Gyr) and metal abundances, but higher helium contents. Fig. 15 shows both hydrogenand helium-burning evolutionary phases for helium-enhanced stellar structures. Note that evolutionary prescriptions for helium-burning structures do not cover, as expected, the temperature range typical of HB stars. At fixed TO age and metal abundance, an increase in the helium content causes a steady decrease in the TO mass. This also means that an increase in helium content also causes the HB morphology to become systematically bluer (hotter, Caloi, Castellani, \& Tornambè 1978; Lee et al. 2005, and references therein). At the same time, the He-core mass becomes smaller, in particular for $\mathrm{Z}=0.0002$ and $\mathrm{Z}=0.001$ it ranges from $0.514 / 0.506(Y=0.23)$, to $0.494 / 0.488(Y=0.33)$, and to $0.479 / 0.474(Y=0.42)$. This means that the extremely hot stellar structures are, at fixed effective temperature, fainter (see Figs. 11 and 15), and in turn, their lifetimes become systematically longer (Castellani et al. 1994; Zoccali et al. 2000). The lifetime of He-enhanced EHB structures when compared with canonical $(Y=0.23)$ structures increases on average by at least $10 \%$ for $Y=0.33$ and $20 \%$ for $\mathrm{Y}=0.42$.

Moreover and even more importantly, the increase in the helium content also causes stellar structures around the TO region $\left(18.65 \leq m_{B} \leq 19.15\right)$ to have significantly shorter lifetimes (see open and filled circles in Fig. 15). In particular, for the metal-poor $(\mathrm{Z}=0.0002)$ chemical composition it ranges from $t_{M S} \sim 1.42$ Gyr for $\mathrm{Y}=0.33$ to $\sim 0.98$ Gyr for $\mathrm{Y}=0.42$, 
i.e., $32 \%$ and $53 \%$ shorter when compared with canonical $(\mathrm{Y}=0.23)$ MS lifetimes. For more metal-rich structures $(\mathrm{Z}=0.001)$ the lifetimes range from $t_{M S} \sim 1.39 \mathrm{Gyr}$ for $\mathrm{Y}=0.33$ to $\sim 0.84$ Gyr for $\mathrm{Y}=0.42$, and the difference with canonical MS lifetimes is $34 \%$ and $60 \%$, respectively. Note that He-enhanced stellar structures present in this magnitude range a very small spread in mass, and indeed $\Delta M \leq 0.005 M_{\odot}$. On the other hand, the evolutionary lifetimes along the RGB are only mildly shorter (see Figs. 11 and 15), and indeed the difference with canonical RG lifetimes is at most of the order of $15 \%$.

The main outcome of the increase in the He content is that the ratio between RG and MS lifetimes increases. Data plotted in the top panel of Fig. 16 show that an increase in He abundance of $\sim 45 \%$ ( $\mathrm{Y}=0.33$ ) causes, in the current metallicity range, at least an increase in the $t(R G) / t(M S)$ ratio of $25 \%$, while an increase of $\sim 85 \%(\mathrm{Y}=0.42)$ gives an increase of more than a factor of two. The predicted lifetime ratios for $\mathrm{Y}=0.42$ and different metal abundances (red and blue dashed lines) attain quite similar values over the entire magnitude range. This behavior is the result of several nonlinear effects. The variations in the metal content and in the He abundance independently affect the evolving mass at fixed stellar age together with the evolutionary times of MS and RGB phases. The predicted ratios are systematically higher than observed. However, predictions for $\mathrm{Z}=0.001$ and $\mathrm{Y}=0.33$ account for the observed ratio, but this would imply that a significant fraction of $\omega$ Cen stars should be metal-rich. This evidence is not supported by current metallicity measurements suggesting a main peak in the metallicity distribution around $[\mathrm{Fe} / \mathrm{H}] \sim-1.7$.

Obviously, these ratios only apply if the entire stellar content of $\omega$ Cen is He-enriched. In the case where $70 \%$ of the stellar populations presents a canonical He content and only $30 \%$ is He-enriched, the predicted ratios are significantly smaller (see bottom panel of Fig. 16) and in excellent agreement with observed ratios. This outcome applies to the two sets of He-enriched models, thus suggesting a marginal dependence on He enhancement. A weak dependence of the stellar luminosity function - below the RGB bump - on helium enhancement has also been recently found by Salaris et al. (2006) using different sets of cluster isochrones accounting for both the helium enhancement and the observed abundance anticorrelations in several heavy elements $(C, N, O, M g, A l)$.

To further constrain this effect we need to estimate the impact of an enhanced He abundance on the ratio of HB stars. Therefore, we use the approach adopted in section 4, to estimate the rate of HB stars. The lifetimes typical of the three HB groups, by accounting for the two different metal abundances and for the same mix of stellar populations are the following:

$$
t_{E B T 1}=0.70 \times t_{E B T 1}(Y=0.23)+0.30 \times t_{E B T 1}(Y=0.33)=0.70 \times[(76+82) \times 0.5]+
$$


$0.30 \times(81+87) \times 0.5] \sim 81 M y r$

$t_{E B T 2}=0.70 \times t_{E B T 2}(Y=0.23)+0.30 \times t_{E B T 2}(Y=0.33)=0.70 \times[(84+90) \times 0.5]+$ $0.30 \times(90+96) \times 0.5] \sim 89 M y r$

$t_{E B T 3}=0.70 \times t_{E B T 3}(Y=0.23)+0.30 \times t_{E B T 3}(Y=0.33)=0.70 \times[(94+101) \times 0.5]+$ $0.30 \times(108+117) \times 0.5] \sim 102 \mathrm{Myr}$

Only the lifetime of the EBT3 group changes for $\mathrm{Y}=0.42$, since the coolest models do not produce HB stars of the EBT1 group and only a negligible fraction of HB stars of the EBT2 group.

$$
\begin{aligned}
& t_{E B T 3}=0.70 \times t_{E B T 3}(Y=0.23)+0.30 \times t_{E B T 3}(Y=0.42)=0.70 \times[(94+101) \times 0.5]+ \\
& 0.30 \times(123+129) \times 0.5] \sim 106 \mathrm{Myr}
\end{aligned}
$$

On the basis of these lifetimes, we can now estimate the rates of HB stars. In particular for $\mathrm{Y}=0.33$ and for star counts based on $B, B-F 625 W$ (ACS) and $B, B-V$ (WFI) CMDs in the innermost annulus $\left(r \leq r_{\alpha}\right)$ we find:

$r(H B, Y=0.33)=N(E B T 3) / 102+N(E B T 2) / 89+N(E B T 1) / 81=349 / 102+98 / 89+$ $798 / 81 \sim 14.43$

while for $\mathrm{Y}=0.42$ we have:

$r(H B, Y=0.42)=N(E B T 3) / 106+N(E B T 2) / 87+N(E B T 1) / 79=349 / 106+98 / 87+$ $798 / 79 \sim 14.51$

The rate of HB stars for the other annuli and for the star counts based on $B, B-F 658 N$ (ACS) and $B, U-V$ (WFI) CMDs are listed in the last two columns of Table 2. Note that an increase in He content causes an increase in the HB lifetime, and in turn a mild decrease in the total rate of $\mathrm{HB}$ stars.

Fig. 17 shows the ratios of $\mathrm{HB}$ and $\mathrm{RG}$ rates in the three different annuli. These ratios were estimated by accounting for a mix of stellar populations with different metal and helium abundances. Interestingly enough, data plotted in the top panel show that the predicted ratios for a mix of $70 \%$ of $\omega$ Cen stars with canonical helium content and $30 \%$ He-enriched $(\mathrm{Y}=0.42)$ are systematically larger than observed. The discrepancy ranges from at least $\sim 15 \%$ to $\sim 25 \%$, and indeed the mean in the three different annuli are $1.248 \pm 0.046$, $1.177 \pm 0.044$, and $1.183 \pm 0.030$. The trend appears similar also for the He-enriched ratios with $\mathrm{Y}=0.33$, but the discrepancy ranges from $\sim 15 \%$ to $\sim 20 \%$, and the mean in the three different annuli are $1.207 \pm 0.030,1.136 \pm 0.049$, and $1.148 \pm 0.018$.

To further constrain the mismatch between theory and observations we decided to com- 
pare the rates of $\mathrm{HB}$ and MS stars. The rates of MS star attain, once again, very similar values, and indeed in the three different annuli star counts based on $B, B-F 625 W$ (ACS) and $B, B-V$ (WFI) CMDs give:

$$
\begin{aligned}
& r(M S)=19932 /(0.7 \times 2093+0.30 \times 1402.5)=10.57 \pm 1.10(Y=0.33) \quad\left(r \leq r_{\alpha}\right) \\
& =19932 /(0.7 \times 2093+0.30 \times 910)=11.47 \pm 1.15(Y=0.42), \\
& r(M S)=19376(0.7 \times 2093+0.30 \times 1402.5)=10.27 \pm 1.03(Y=0.33) \quad\left(r_{\alpha}<r \leq r_{\beta}\right) \\
& =19376 /(0.7 \times 2093+0.30 \times 910)=11.15 \pm 1.10(Y=0.42),
\end{aligned}
$$

and

$$
\begin{gathered}
r(M S)=15223 /(0.7 \times 2093+0.30 \times 1402.5)=8.07 \pm 0.81(Y=0.33) \quad\left(r>r_{\beta}\right) \\
=15223 /(0.7 \times 2093+0.30 \times 910)=8.76 \pm 0.88(Y=0.42) .
\end{gathered}
$$

At the same time, the semi-empirical ratios given by star counts based on $B, B-F 658 N$ (ACS) and $B, U-V(\mathrm{WFI}) \mathrm{CMDs}$ give:

$$
\begin{aligned}
& r(M S)=19935 /(0.7 \times 2093+0.30 \times 1402.5)=10.57 \pm 1.10(Y=0.33) \quad\left(r \leq r_{\alpha}\right) \\
& =19935 /(0.7 \times 2093+0.30 \times 910)=11.47 \pm 1.15(Y=0.42), \\
& r(M S)=19815 /(0.7 \times 2093+0.30 \times 1402.5)=10.51 \pm 1.05(Y=0.33) \quad\left(r_{\alpha}<r \leq r_{\beta}\right) \\
& =19815 /(0.7 \times 2093+0.30 \times 910)=11.40 \pm 1.14(Y=0.42),
\end{aligned}
$$

and

$$
\begin{gathered}
r(M S)=14187 /(0.7 \times 2093+0.30 \times 1402.5)=7.52 \pm 0.75(Y=0.33) \quad\left(r>r_{\beta}\right) \\
=14187 /(0.7 \times 2093+0.30 \times 910)=8.16 \pm 0.81(Y=0.42) .
\end{gathered}
$$

Therefore, the total rate of MS stars in the selected box is $\sim 28$ stars per Myr for $\mathrm{Y}=0.33$ and $\sim 30$ stars per Myr for $\mathrm{Y}=0.42$. Hence, the discrepancy between lifetimes and star counts is of the order of $30 \%$ for $\mathrm{Y}=0.33$ and of the order of $24 \%$ for $\mathrm{Y}=0.42$. Note that the above rates have been estimated by assuming, at fixed helium content, the mean HB and MS lifetimes for the two different metal abundances $(\mathrm{Z}=0.0002, \mathrm{Z}=0.001)$. Current findings indicate that a mix of stellar populations comprising $70 \%$ with canonical $\mathrm{He}(\mathrm{Y}=0.23)$ and $30 \%$ with He-enhanced $(Y=0.33$ or $Y=0.42$ ) stars does not account for the observed excess of HB stars in $\omega$ Cen.

In passing we note that the $\mathrm{HB} / \mathrm{MS}$ ratio presents a twofold advantage when compared with the $\mathrm{RG} / \mathrm{MS}$ and the $\mathrm{HB} / \mathrm{RG}$ ratio: i) Current predictions indicate that an increase in He abundance causes a decrease in the lifetime of both RG and MS stars, whereas the lifetime 
of hot $\mathrm{HB}$ stars increases. Moreover, the HB/MS ratio has the largest sensitivity to changes in He abundance. ii) The HB/MS ratio is marginally affected by field star contamination $($ see $\S 4)$.

\section{Discussion and final remarks}

We have presented an extensive photometric investigation on Horizontal Branch, Red Giant Branch and Main Sequence stars in the Galactic globular cluster $\omega$ Cen. The central regions of the cluster were covered with a mosaic of $F 435 W, F 625 W$, and $F 658 N$-band data collected with ACS on board the Hubble Space Telescope. The outer reaches were covered with a large set of $U, B, V, I$-band data collected with the mosaic camera, WFI, available at the $2.2 \mathrm{~m}$ ESO/MPI telescope. The current photometric data cover more than $99 \%$ of the total flux of the cluster and the final catalogue includes approximately 1.7 million stars. This unprecedented photometric catalogue allowed us to identify more than 3,200 likely HB stars, the largest sample collected in a globular cluster to date, and more than 12,500 stars brighter than the base of the RGBs and fainter than the RGB bumps $(15 \leq B \leq 18)$. The sizable sample of HB stars allowed us to constrain on a quantitative basis the change in the HB morphology as a function of the radial distance. The relative number of extreme HB stars decreases from $\sim 30 \%$ to $\sim 21 \%$ when moving from the center toward the outer reaches of the cluster. On the other hand, the fraction of less hot HB stars (EBT1) increases from $\sim 62 \%$ to $\sim 72 \%$.

Two physical mechanisms that might partially account for the discrepancy between observed and predicted star counts are the so-called "breathing pulses" (Castellani et al. 1985) and mild convective core overshooting. These mechanisms increase the central Heburning lifetime by roughly the $15 \%$ (Caputo et al. 1989; Cassisi et al. 2001; Straniero et al. 2003). On the other hand, stellar rotation has an opposite trend because the rotation delays the He core flash at the tip of the RGB. This causes an increase in the mass of the He core and a decreases in the envelope mass (Renzini 1977), since the mass loss is more efficient close to the tip of the RGB. Therefore, rotating HB structures are typically bluer and brighter than "canonical" HB stars. Moreover, one should also take into account the rotationally induced mixing along the RGB phase (Denissenkov \& VandenBerg 2003; Palacios et al. 2006). If the envelope mixing does not reach the edge of the H-burning shell the effect on HB models is negligible. On the contrary, if the mixing reaches the H-burning shell, then the He abundance in the envelope of bright RGB structures increases, thus causing a higher luminosity and a larger mass loss close to the tip of RGB. The aftermath of such a mechanism is a brighter and bluer HB morphology (see e.g. Sweigart \& Catelan 1998, and 
references therein). The rotational HB models present, when compared with canonical ones, larger He core masses, and in turn more luminous stellar structures. This means that the HB lifetime of the rotational HB models is, at fixed effective temperature, shorter than for canonical ones. Moreover, empirical evidence suggests that the projected rotational velocity among hot HB stars $\left(T_{e}>10,000 \mathrm{~K}\right)$, in several GCs that show a well-defined Blue Tail, is quite constant and smaller than $10 \mathrm{~km} / \mathrm{sec}$ (see Fig. 5 in Recio Blanco et al. 2004), while it presents a mild increase only among cooler $\left(T_{e} \leq 9,000 \mathrm{~K}\right)$ HB stars (Behr et al. 2000).

This evidence might suggest that the higher density in the central regions plays a crucial role in the formation of extreme HB stars. However, $\omega$ Cen presents a central density that is at least one order of magnitude smaller $\left(\log \rho_{0}=3.12 L_{\odot} p c^{-3}\right)$ than other massive GGCs (47 Tuc, NGC 6397). Therefore, it might be interesting to estimate the expected frequency of stellar collisions for this very massive GC. It was demonstrated by King, Surdin \& Rastorguev (2002) that the number of stellar collisions per year for a GC with a King profile is given by $\Gamma_{c}=5 \times 10^{-15}\left(\Sigma_{0}^{3} \times r_{c}\right)^{1 / 2}$, where $r_{c}$ is the core radius in parsec and $\Sigma_{0}$ is the central surface brightness in units of $L_{\odot, V} p c^{-2}$. To estimate the $r_{c}$ in parsec we adopted the true distance modulus $(\mu=13.70 \pm 0.6 \pm 0.6)$ recently provided by Del Principe et al. (2006) and the core radius $\left(r_{c} \sim 2.6\right.$ arcmin) provided by Trager et al. (1995) and more recently by Ferraro et al. (2006). We find $r_{c}=4.13 \mathrm{pc}$, and in turn that $\Gamma_{c}$, i.e. the number of collisions is $5.85 \times 10^{-9} y^{-1}$. Following the assumptions adopted by Piotto et al. (2004) in order to estimate the probability that a single star undergoes a stellar collision in $1 \mathrm{yr}, \Gamma_{\star}$, we divided $\Gamma_{c}$ by the number of cluster stars. In order to estimate $N_{\star}$ we adopted the mass-to-light ratio $\left(M / L_{V} \sim 2.5\right)$ recently estimated by van de Ven et al. (2006), the absolute visual magnitude of $\omega$ Cen is $M_{V}=-10.39\left(V_{t}=3.68, E(B-V)=0.12\right.$, Harris 1996) and a typical mass of $0.4 M_{\odot}$. The total number of stars we find is $7.74 \times 10^{6}$. This estimate agrees quite well with the dynamical estimate of the total mass of the cluster provided by van de Ven et al. (2006). They found $M_{t}=2.5 \times 10^{6} M_{\odot}$ that assuming the same typical mass provides a number of stars in $\omega$ Cen of $6.25 \times 10^{6}$. Therefore, the probability that a single star centrally located experiences a collision in $1 \mathrm{yr}$ is $\log \Gamma_{\star}=-15.12$. The current collision rate is approximately one order of magnitude smaller than the collision rate in $\operatorname{NGC2808}\left(\log \Gamma_{\star}=-14.05\right)$ a relatively massive GC $\left(M_{V}=-9.36\right.$, Harris 1996) characterized by a higher central density $\left(\log \rho_{0}=4.61 L_{\odot} p c^{-3}\right)$ and a HB morphology including a significant number of red HB stars and very extended blue HB tail (Castellani et al. 2006a). However, the current collision rate in $\omega$ Cen is slightly higher than the collision rate of $\mathrm{NGC} 4833\left(\log \Gamma_{\star}=-15.31\right)$ a GC with a central density $\left(\log \rho_{0}=3.06 L_{\odot} p c^{-3}\right)$ and a HB morphology (Melbourne et al. 2000) quite similar to $\omega$ Cen. Current findings seem to support the evidence brought forward by Castellani et al. (2006a) that the Blue Tails, if affected by cluster dynamics, should be considered more a transient phenomenon rather than an intrinsic feature of GCs. We plan 
to address this topic on the basis of a larger sample of GCs in a forthcoming paper.

The referee suggested two different working hypotheses that might account for the peculiar radial distribution of $\mathrm{HB}$ stars.

i) Let us assume that extreme HB stars are the progeny of WD binaries. This would imply that the radial gradient of HB stars could be the aftermath of mass segregation causing a larger fraction of binaries to be located in the cluster center. Recent empirical evidence indicates that more massive GCs tend to have a larger number of HB stars and bluer HB morphologies (Davies et al. 2004; Recio Blanco et al. 2006). However, Ferraro et al. (2006) found a lack of mass segregation in $\omega$ Cen , since the radial distribution of Blue Stragglers does not appear to be centrally peaked. These objects are thought to be the evolution of binary systems and their radial distribution in GCs is typically more concentrated than single cluster stars.

ii) Let us assume that a radial gradient in the initial He abundance was already present at the epoch of the cluster formation. Could this initial gradient still persist due to the long two-body relaxation time of $\omega$ Cen ? This working hypothesis is supported by the empirical evidence that more metal-rich cluster stars are more centrally concentrated than metal-poor ones (Norris et al. 1996, 1997). Moreover, Sollima et al. (2007) found that stars belonging to the so-called "blue-MS" are more centrally concentrated than stars belonging to the "red-MS". These findings together with the evidence that stars in the "blue-MS" appear to be less metal-poor (Piotto et al. 2005) and that the two body relaxation time increases outside the half-mass radius suggest that a radial gradient in He abundance may have been set up at the epoch of cluster formation. However, recent chemical evolution models might account for the metallicity distribution in $\omega$ Cen, but do not account for the significant He enhancement suggested to explain the "blue-MS" (Romano et al. 2007).

To provide firm constraints on the stellar populations in $\omega$ Cen we performed detailed comparisons of large samples of evolved and MS stellar tracers with homogeneous evolutionary prescriptions. In particular, the comparison between the observed star counts (RG, HB, MS) and the theoretical framework based on a canonical helium content ( $\mathrm{Y}=0.23)$ brings forward several interesting findings.

i) Star counts along the $R G B$ - Evolutionary lifetimes for stellar structures along the RGB phase constructed by adopting a broad range of metal abundances $(\mathrm{Z}=0.0002,0.001)$, different stellar masses $\left(M=0.80,0.85 M_{\odot}\right)$ agree quite well with empirical RG star counts fainter the RGB Bump.

ii) Stellar population ratios- The comparison between theory and observations discloses that the observed ratio of $\mathrm{HB}$ and RG stars is systematically larger $(30 \%-40 \%)$ than the ratio of $\mathrm{HB}$ and $\mathrm{RG}$ lifetimes predicted by evolutionary models with metal abundances 
$(\mathrm{Z}=0.0002, \mathrm{Z}=0.001)$ that bracket the observed spread in metallicity of $\omega$ Cen stars.

iii) The ratio between $H B, R G$, and $M S$ stars- The comparison between the observed ratio of $\mathrm{RG}$ and MS stars with the predicted ratio of their evolutionary lifetimes is suggestive of a mild excess of RG stars in the faint region of the RGB. By using evolutionary tracks with canonical compositions, the discrepancy ranges once again from 10 to 15\%. The same outcome applies to the observed ratio between HB and MS stars. However, their excess when compared with the predicted ratio is of the order of $43 \%$. This circumstantial evidence suggests the possibility that a fraction of bright RG stars miss the central helium flash evolutionary phase.

Recent empirical evidence concerning the occurrence in $\omega$ Cen of a blue MS and of a well-defined blue HB tail have been explained with the presence of a He-enhanced stellar population. To constrain this appealing working hypothesis we constructed several evolutionary tracks and HB evolutionary models using the same metallicity range but higher He contents, namely $\mathrm{Y}=0.33$ and $\mathrm{Y}=0.42$. The comparison between theory and observations was performed by assuming a mix of stellar populations made, according to empirical evidence, with $70 \%$ of canonical stars and 30\% He-enhanced stars. We found the following results.

i) The ratio between $R G, H B$, and $M S$ stars- The observed ratio of RG and MS stars agrees quite-well with the predicted lifetimes of mixed He-enhanced ( $\mathrm{Y}=0.33$ or $\mathrm{Y}=0.42$ ) stellar populations. Thus suggesting a negligible sensitivity of this ratio on the He content. On the other hand, the observed HB and RG star counts are still 15\%-25\% larger than predicted for He-mixed stellar populations with $(\mathrm{Y}=0.42)$, but the discrepancy is on average a factor of two smaller than for predicted ratios based on evolutionary models with canonical He abundance. The same outcome applies to the predicted ratio for $\mathrm{Y}=0.33$ but the discrepancy ranges from $\sim 15 \%$ to $\sim 20 \%$. Moreover and even more importantly, the observed ratios between HB and MS star counts are once again from $\sim 24 \%$ and $\sim 30 \%$ larger than predicted for He-mixed populations with $\mathrm{Y}=0.42$ and $\mathrm{Y}=0.33$, respectively.

ii) New empirical constraints- The findings based on standard and He-mixed stellar populations have quite different impacts on the final evolutionary fate of low-mass stars. The theoretical scenario based on a canonical helium content also implies that a fraction of RG stars does not approach the tip of the RGB, missing the core-helium flash phase, and might end up their evolution either as extreme HB stars or as He-core WDs (Castellani et al. 2006b). Therefore, we should also observe an excess of AGB-manquè stars and of bright WDs. The theoretical framework based on He-mixed stellar populations is puzzling, and indeed the ratios between HB and MS stars is roughly a factor of two larger than the 
excess between HB and RG stars. However, the ratios between MS and RG star counts agree quite-well with the ratio of evolutionary lifetimes.

During the last few years several homogeneous photometric investigations covering a substantial fraction of the body of GGCs found mounting evidence for peculiar radial distributions among different stellar tracers (Castellani et al. 2006a; Sollima et al. 2007). Moreover, a significant excess of RG stars above and below the RGB bump has also been detected in several GCs (Sandquist et al. 1999; Pollard et al. 2005). On the other hand, in a very recent investigation Sandquist \& Martel (2006) found a well-defined deficiency of RG stars along the RGB of the massive GGC NGC2808. This globular shows an extended blue HB tail and the authors also suggest that strong mass loss events might account for such an empirical evidence. An accurate and quantitative analysis of RG stars in $\omega$ Cen across and above the RGB bump is mandatory to constrain the efficiency of this possible evolutionary chanel. It goes without saying that the new mosaic CCD cameras with FOV of the order of 1 square degree might play a crucial role to provide sound quantitative constraints on these new compelling features.

It is a pleasure to thank S. Cassisi for several discussions and a detailed reading of an early draft of this manuscript. We also thank F. De Angeli for many enlightening suggestions and insights concerning the collisional rate of GCs. We acknowledge an anonymous referee for his/her positive and pertinent comments that helped us to improve the readability of the manuscript. This work was partially supported by PRIN-INAF2005 (P.I.: A. Buzzoni), "Galactic Stellar Populations", by PRIN-INAF2004 (P.I.: M. Bellazzini), "A hierarchical merging tale told by stars: motions, ages and chemical compositions within structures and substructures of the Milky Way", and by Particle Physics and Astronomy Research Council (PPARC). We also thank the ESO and the HST Science Archive for their prompt support. This publication makes use of data products from VizieR (Ochsenbein, Bauer, \& Marcout 2000) and from the Two Micron All Sky Survey, which is a joint project of the University of Massachusetts and the Infrared Processing and Analysis Center/California Institute of

Technology, funded by the National Aeronautics and Space Administration and the National Science Foundation.

\section{REFERENCES}

Anderson, J. 2002, in Omega Centauri: A Unique Window into Astrophysics, ed. F. van Leeuwen, J. Hughes, \& G. Piotto (San Francisco: ASP), 265, 87

Baade, D. et al. 1999, The Messenger, 95, 15 
Bailyn, C.D., Sarajedini, A., Cohn, H., Lugger, P.M., \& Grindlay, J.E. 1992, AJ, 103, 1564

Bedin, L. R., Piotto, G., Zoccali, M. et al. 2000, A\&A, 363, 159

Bedin, L. R., Piotto, G., Anderson, J., Cassisi, S. et al. 2004, ApJ, 605, 125

Behr, B.B., Cohen, J.G., McCarthy, J.K. 2000, ApJ, 531, L37

Bekki, K., \& Norris, J. E. 2006, ApJ, 637, L109

Bessel, M. S., Castelli, F. \& Plez, B. 1998, A\&A, 333, 231

Bono G., Castellani V., Degl'Innocenti S., Pulone, L. 1995, A\&A 297, 115

Brown, T.M.,Sweigart, A.V., Lanz T., Landsman, W.B., \& Hubeny, I. 2001, ApJ, 562, 368

Calamida, A. et al. 2005, ApJ, 634, 69

Caloi, V., Castellani, V., \& Tornambè, A. 1978, A\&AS, 33, 169

Caputo, F., Chieffi, A., Tornambe, A., Castellani, V., Pulone, L. 1989, ApJ, 340,241

Caputo F., Cassisi S. 2002, MNRAS, 333, 825

Cardelli, J.A., Clayton, G.C., \& Mathis, J.S. 1989, ApJ, 345, 245

Cariulo, P., Degl'Innocenti, S., \& Castellani, V. 2004, A\&A, 424, 927

Cassisi, S., Castellani, V., Degl'Innocenti, S. \& Weiss, A. 1998, A\&AS, 129, 267

Cassisi, S. et al. 2001, A\&A 366, 578

Cassisi, S., Schlattl, H., Salaris, M., \& Weiss, A. 2003, ApJ, L43

Castellani, V., Chieffi, A., Pulone, L., \& Tornambé, A. 1985, ApJ, 296, 204

Castellani, M., \& Castellani, V. 1993, ApJ, 407, 649

Castellani, M., Castellani, V., Pulone, L., \& Tornambè, A. 1994, A\&A, 282, 771

Castellani, V., Iannicola, G., Bono, G., Zoccali, M., Cassisi, S., \& Buonanno, R. 2006a, A\&A, 446, 569

Castellani, M., Castellani, V., \& Prada Moroni, P. G. 2006b, A\&A, 457, 569

Castelli, F. \& Kurucz, R.L. 2003, in IAU Symposium 210, ed. N. Piskunov, W.W. Weiss, \& D.F. Gray,(Uppsala, Sweden), 20 
Chieffi, A., Straniero, O. 1989, ApJS, 71, 47

Ciacio, F., Degl'Innocenti, S., Ricci, B. 1997, A\&AS, 123, 449

Corsi, C.E. et al. 2003, MmSAI, 74, 884

Cutri, R. M. \& 2MASS collaboration 2003, www.ipac.caltech.edu/2mass

Da Costa, G. S., Villumsen, J. V. 1981, in IAU. Coll. 68, Astrophysical Parameters for Globular Clusters, (Knudsen), 527

Da Costa, G.S., Norris, J., Villumsen, J.V. 1986, ApJ, 308, 743

D’Antona, F., Bellazzini, M., Caloi, V., Fusi Pecci, F., Galleti, S., \& Rood, R. T. 2005, ApJ, 631,868

Davies, M. B., Piotto, G., de Angeli, F. 2004, MNRAS, 349, 129

D’Cruz, N.L., Dorman, B., Rood, R.T., O’Connel, R.W. 1996, ApJ, 466, 359

D'Cruz, N.L., Rood, R.T., O'Connel, R.W. et al. 2002, in Omega Centauri: A Unique Window into Astrophysics, ed. F. van Leeuwen, J. Hughes, \& G. Piotto (San Francisco: ASP), 235

Del Principe, M. et al. 2006, ApJ, 77, 330

Ferraro, F. R., Sollima, A., Rood, R. T., Origlia, L., Pancino, E., Bellazzini, M. 2006, ApJ, 638,433

Denissenkov, P. A., VandenBerg, Don A. 2003, ApJ, 598, 1246

Freyhammer, L.M. et al. 2005, ApJ, 623, 860

Gilmore G., King I., van der Kruit P.C. 1990, in Saas-Fee advanced course, The Milky Way as a galaxy, ed. Buser R. \& King I., (Geneva), 19

Harris, W.E. 1996, AJ, 112, 1487

Hilker, M., Kayser, A., Richtler, T., \& Willemsen, P. 2004, A\&A, 422, L9

Iben, I. Jr. 1991, ApJS, 76, 55

Iglesias, C.A., Rogers, F.J. 1996, ApJ, 464, 943

Ivanova, N., Heinke, C. O., Rasio, F. A., Taam, R. E., Belczynski, K., \& Fregeau, J. 2006, MNRAS, 372, 1043 
Jordi, K., Grebel, E. K., \& Ammon, K. 2006, A\&A, accepted, astro-ph/0609121

Kaluzny, J., Thompson, I., Krzeminski, W., Olech, A., Pych, W., \& Mochejska, B. 2002, in Omega Centauri: A Unique Window into Astrophysics, ed. F. van Leeuwen, J. Hughes, \& G. Piotto (San Francisco: ASP), 155

King, I. 1962, AJ, 67, 471

King, A. R., Surdin, V. G., \& Rastorguev, A. S. 2002, Vvedenie v Klassiceskuju Zvezdnuju Dinamiku (Moskva: Editorial URSS)

Lee, Y.-W. et al. 2005, AJ, 621, L57

Lub, J. 2002, in Omega Centauri: A Unique Window into Astrophysics, ed. F. van Leeuwen, J. Hughes, \& G. Piotto (San Francisco: ASP), 95

Maeder, A., \& Meynet, G. 2006, A\&A, 448, L37

Melbourne, J., Sarajedini, A., Layden, A., Martins, D. H. 2000, AJ, 120, 3127

Meylan, G., Mayor, M., Duquennoy, A., \& Dubath, P. 1995, A\&A, 303, 761

Moehler, S., Sweigart, A. V., Landsman, W. B., Dreizler, S. 2002, A\&A, 395, 37

Moehler, S., \& Sweigart, A. V. 2006, A\&A, 455, 943

Momany, Y., et al. 2004, A\&A, 420, 605

Monelli, M. et al. 2003, AJ, 126, 218

Monelli, M., Corsi, C.E., Castellani, V. et al. 2005, ApJ, 621, L117

Norris, J. E. 2004, ApJ, 612, 25

Norris, J. E., Freeman, K. C., Mayor, M., \& Seitzer, P. 1997, ApJ, 487, L187

Norris, J. E., Freeman, K. C., \& Mighell, K. J. 1996, ApJ, 462, 241

Ochsenbein, F., Bauer, P., \& Marcout, J. 2000, A\&AS, 143, 221

Palacios, A., Charbonnel, C., Talon, S., Siess, L. 2006, A\&A, 453, 261

Pancino, E., Ferraro, F. R., Bellazzini, M., Piotto, G., Zoccali, M. 2000, ApJ, 534, 83

Pancino, E., Pasquini, L., Hill, V., Ferraro, F.R., \& Bellazzini, M. 2002, ApJL, 568, L101 
Pietrinferni, A., Cassisi, S., Salaris, M. and Castelli, F. 2004, ApJ, 612, 168

Pietrinferni, A., Cassisi, S., Salaris, M. and Castelli, F. 2006, ApJ, 642, 697

Piotto, G., et al. 2004, ApJ, 604, L109

Piotto, G. et al. 2005, ApJ, 621, 777

Pollard, D. L., Sandquist, E. L., Hargis, J. R., Bolte, M. 2005, ApJ, 628, 729

Recio-Blanco, A., Aparicio, A., Piotto, G., de Angeli, F., Djorgovski, S. G 2006, A\&A, 452, 875

Recio-Blanco, A., Piotto, G., Aparicio, A., Renzini, A. 2004, A\&A, 417, 597

Renzini, A. 1977, in The Evolution of Population II Stars and Mass Loss and Stellar Evolution, ed. P. Bouvier and A. Maeder, (Sauverny, Switzerland), 151

Rey, S-C. et al. 2000, AJ, 119, 1824

Rey, S-C., Joo, J-M., Sohn, Y-J., Ree C-H., Lee, Y-W. 2002, in Omega Centauri: A Unique Window into Astrophysics, ed. F. van Leeuwen, J. Hughes, \& G. Piotto (San Francisco: ASP), 177

Romano, D., et al. 2007, MNRAS, accepted, astro-ph/0701162

Salaris, M., Weiss, A., Ferguson, J. W., \& Fusilier, D. J. 2006, ApJ, 645, 1131

Salaris, M.; Riello, M.; Cassisi, S.; Piotto, G. 2004, A\&A, 420, 911

Sandquist, E. L., Bolte, M., Langer, G. E., Hesser, J. E., \& Mendes de Oliveira, C. 1999, ApJ, 518, 262

Sandquist, E. L. \& Martel, A. R. 2006, ApJ Letters, accepted, astro-ph/0611278

Sirianni, M., Jee, M. J., Benatez, N., et al. 2005, PASP, 117, 1049

Sollima, A., Pancino, E., Ferraro, F. R., Bellazzini, M., Straniero, O., \& Pasquini, L. 2005, ApJ, 634, 332

Sollima, A., Borissova, J., Catelan, M., Smith, H. A., Minniti, D., Cacciari, C., \& Ferraro, F.R. 2006, AJ, 640, L43

Sollima, A., Ferraro, F. R., Bellazzini, M., Origlia, L., Straniero, O., \& Pancino, E., 2007, ApJ, 654, 915 
Spergel, D. N. WMAP collaboration, ApJ, submitted, astro-ph/0603449

Stetson, P. B. 1991, in The formation and evolution of star clusters, ed. K. Janes, (San Francisco: ASP), 88

Stetson, P. B. 2000, PASP, 112, 925

Stetson, P. B., Bruntt, H., \& Grundahl, F. 2003, PASP, 115, 413

Straniero, O., Dominguez, I., Imbriani, G., Piersanti, L. 2003, ApJ, 583, 878

Suntzeff, N. B., \& Kraft, R. P. 1996, AJ, 111, 1913

Sweigart, A. V. 1997, in Third Conference on Faint Blue Stars, ed. A.G.D. Phillip, J. Liebert, \& R.A. Saffer (Schenrctaday: L.Davis Press), 3

Sweigart, A. V., Catelan, M. 1998, ApJ, 501, L63

Thoul, A., Bahcall, J., Loeb, A. 1994, ApJ, 421, 828

Trager, S. C., King, I. R., \& Djorgovski, S. 1995, AJ, 109, 218

van de Ven, G., van den Bosch, R. C. E., Verolme, E. K., \& de Zeeuw, P. T. 2006, A\&A, 445,513

van Leeuwen, F., Le Poole, R. S., Reijns, R. A., Freeman, K. C., \& de Zeeuw, P. T. 2000, A\&A, 360, 472

Walker, A. R. 1994, PASP, 106, 828

Weldrake, D. T. F., Sackett, P. D. \& Bridges, T. J. 2006, AJ, accepted, astro-ph/0610704

Whitney, J.H., O’Connell, R.W., Rood, R.T. et al. 1994, AJ, 108, 1350

Whitney, J.H., Rood R.T., O’Connell R.W et al. 1998, ApJ, 495, 284

Yasuda, N., \& SDSS collaboration 2001, AJ, 122, 1104

Zoccali, M., Cassisi, S., Bono, G., Piotto, G., Rich, R. M., \& Djorgovski, S. G. 2000, ApJ, 538,289

Zoccali, M., \& Piotto, G. 2000, A\&A, 358, 943 
Table 1. Log of scientific CCD images of Omega Centauri collected with the WFI available at the $2.2 \mathrm{~m} \mathrm{ESO/MPI} \mathrm{telescope.}{ }^{\mathrm{a}}$

\begin{tabular}{|c|c|c|c|c|c|c|}
\hline $\begin{array}{c}\text { Frame }^{\mathrm{b}} \\
\text { (1) }\end{array}$ & $\begin{array}{l}\mathrm{RA}^{\mathrm{c}} \\
(2)\end{array}$ & $\begin{array}{l}\mathrm{DEC}^{\mathrm{d}} \\
(3)\end{array}$ & $\begin{array}{l}\text { MJD }^{\mathrm{e}} \\
(4)\end{array}$ & $\begin{array}{c}\text { Exposure }^{\mathrm{f}} \\
(5)\end{array}$ & $\begin{array}{c}\text { Filter }^{\mathrm{g}} \\
(6)\end{array}$ & $\begin{array}{l}\text { Seeingh } \\
(7)\end{array}$ \\
\hline WFI.1999-01-24T08:18:52.934 & $13: 26: 18.9$ & $-47: 36: 01.1$ & 51202.3464460 & 29.92 & $\mathrm{~B}$ & 1.11 \\
\hline WFI.1999-01-24T08:21:57.925 & $13: 26: 42.7$ & $-47: 36: 01.5$ & 51202.3485871 & 29.92 & $\mathrm{~B}$ & 0.91 \\
\hline WFI.1999-01-24T08:24:01.313 & $13: 26: 48.6$ & $-47: 36: 01.8$ & 51202.3500152 & 29.92 & $\mathrm{~B}$ & 1.00 \\
\hline WFI.1999-01-24T08:25:44.311 & $13: 26: 48.7$ & $-47: 36: 01.5$ & 51202.3512073 & 299.92 & B & 0.98 \\
\hline WFI.1999-03-25T05:28:39.461 & $13: 26: 44.8$ & $-47: 26: 34.2$ & 51262.2282345 & 149.92 & I & 0.84 \\
\hline WFI.1999-03-25T05:32:42.409 & $13: 26: 47.8$ & $-47: 26: 03.8$ & 51262.2310464 & 149.92 & I & 0.75 \\
\hline WFI.1999-03-25T05:36:40.398 & $13: 26: 50.8$ & $-47: 25: 34.4$ & 51262.2338009 & 149.92 & I & 0.73 \\
\hline WFI.1999-03-25T06:04:59.817 & $13: 26: 44.8$ & $-47: 26: 34.5$ & 51262.2534701 & 199.92 & $\mathrm{~V}$ & 0.86 \\
\hline WFI.1999-03-25T06:10:25.156 & $13: 26: 47.8$ & $-47: 26: 04.6$ & 51262.2572356 & 199.92 & $\mathrm{~V}$ & 0.95 \\
\hline WFI.1999-03-25T06:14:59.839 & $13: 26: 50.7$ & $-47: 25: 33.7$ & 51262.2604148 & 199.92 & $\mathrm{~V}$ & 1.02 \\
\hline WFI.1999-04-29T00:19:41.952 & $13: 26: 46.4$ & $-47: 35: 48.5$ & 51297.0136800 & 89.92 & I & 0.59 \\
\hline WFI.1999-04-29T00:23:20.786 & $13: 26: 46.4$ & $-47: 31: 38.8$ & 51297.0162128 & 89.92 & I & 0.59 \\
\hline
\end{tabular}

a Table 1 is available in its entirety via the link to the machine-readable version above. A portion is shown here for guidance regarding its form and content.

${ }^{\mathrm{b}}$ Frame identification.

${ }^{\mathrm{c}}$ Right ascension (J2000) in units of hours, minutes, and seconds.

${ }^{\mathrm{d}}$ Declination (J2000) in units of degrees, arcminutes, and arcseconds.

e Modified Julian Date (JD - 2400000.5) at the start of the exposure.

${ }^{\mathrm{f}}$ Exposure time (sec).

gFilter name. $U$-band and $B$-band data collected in 1999 and 2000 observing runs adopted the filters $U / 38(E S O \# 841)$ and $B / 99(E S O \# 842)$, while subsequent data have been collected with the new filters $U / 50(E S O \# 877)$ and $B / 123(E S O \# 878)$.

${ }^{\mathrm{h}}$ Seeing of individual images $(\operatorname{arcsec})$. 
Table 2. The number of HB stars in the three selected HB subgroups detected in the three different regions of the cluster. Figures within brackets give for each region the relative fraction of the different subgroups with respect to the total number of HB stars.

\begin{tabular}{|c|c|c|c|c|c|c|c|c|}
\hline Radius & & $\mathrm{N}(\mathrm{EBT} 1)$ & $\mathrm{N}(\mathrm{EBT} 2)$ & $\mathrm{N}(\mathrm{EBT} 3)$ & $N_{t o t}$ & $r(H B)^{\mathrm{a}}$ & $r(H B)^{\mathrm{b}}$ & $r(H B)^{\mathrm{c}}$ \\
\hline \multicolumn{9}{|c|}{ Star counts based on $B, B-F 625 W$ (ACS) and $B, B-V$ (WFI) CMDs } \\
\hline$r \leq r_{\alpha}$ & & $798(64 \pm 3 \%)$ & $98(8 \pm 1 \%)$ & $349(28 \pm 2 \%)$ & 1245 & 14.79 & 14.43 & 14.51 \\
\hline$r_{\alpha}<r \leq r_{\beta}$ & $402^{\mathrm{d}}$ & $+293^{\mathrm{e}}(60 \pm 3 \%)$ & $56^{\mathrm{d}}+40^{\mathrm{e}}(8 \pm 1 \%)$ & $235^{\mathrm{d}}+136^{\mathrm{e}}(32 \pm 2 \%)$ & 1162 & 13.69 & 13.34 & 13.39 \\
\hline$r>r_{\beta}$ & & $650(72 \pm 4 \%)$ & $64(7 \pm 1 \%)$ & $190(21 \pm 2 \%)$ & 904 & 10.90 & 10.65 & 10.75 \\
\hline Total & & $2143(65 \pm 2 \%)$ & $278(8 \pm 1 \%)$ & $910(27 \pm 1 \%)$ & 3311 & 39.38 & 38.42 & 38.64 \\
\hline \multicolumn{9}{|c|}{ Star counts based on $B, B-F 658 N(\mathrm{ACS})$ and $B, U-V$ (WFI) CMDs } \\
\hline$r \leq r_{\alpha}$ & & $787(64 \pm 3 \%)$ & $99(8 \pm 1 \%)$ & $349(28 \pm 2 \%)$ & 1235 & 14.66 & 14.30 & 14.38 \\
\hline$r_{\alpha}<r \leq r_{\beta}$ & $404^{\mathrm{d}}$ & $+299^{\mathrm{e}}(61 \pm 3 \%)$ & $59^{\mathrm{d}}+40^{\mathrm{e}}(9 \pm 1 \%)$ & $237^{\mathrm{d}}+110^{\mathrm{e}}(30 \pm 2 \%)$ & 1149 & 13.58 & 13.24 & 13.30 \\
\hline$r>r_{\beta}$ & & $618(72 \pm 4 \%)$ & $61(7 \pm 1 \%)$ & $181(21 \pm 2 \%)$ & 860 & 10.37 & 10.13 & 10.23 \\
\hline Total & & $2108(65 \pm 2 \%)$ & $269(8 \pm 1 \%)$ & $877(27 \pm 1 \%)$ & 3244 & 38.61 & 37.67 & 37.90 \\
\hline
\end{tabular}

${ }^{a}$ Rate of HB stars, i.e., the number of HB stars formed per Myr according to the lifetime of the three different EBT groups. The lifetimes were estimated as the mean of HB models with $\mathrm{Z}=0.0002(\mathrm{Y}=0.23)$ and $\mathrm{Z}=0.001(\mathrm{Y}=0.232)$.

${ }^{\mathrm{b}}$ Rate of HB stars, i.e., the number of HB stars formed per Myr according to the lifetime of the three different EBT groups. The lifetimes were estimated by accounting for a mix of stellar populations with different metal $(\mathrm{Z}=0.0002,0.001)$ and helium $(\mathrm{Y}=0.23,0.33)$ abundances.

${ }^{\mathrm{c}}$ Rate of HB stars, i.e., the number of HB stars formed per Myr according to the lifetime of the three different EBT groups. The lifetimes were estimated by accounting for a mix of stellar populations with different metal $(\mathrm{Z}=0.0002,0.001)$ and helium $(\mathrm{Y}=0.23,0.42)$ abundances.

${ }^{\mathrm{d}}$ Star counts based on ACS data $\left(r_{\alpha}<r \leq r_{a c s}\right)$.

e Star counts based on WFI data $\left(r_{a c s}<r \leq r_{\beta}\right)$. 
Table 3. The number of RG stars detected in the three selected cluster regions. Figures within brackets give for each region the relative fraction with respect to the total number of RG stars.

\begin{tabular}{lrrrr}
\hline \hline \multicolumn{1}{c}{ Radius } & \multicolumn{1}{c}{$R G^{\mathrm{a}}$} & \multicolumn{1}{c}{$\omega 3^{\mathrm{a}}$} & \multicolumn{1}{c}{$R G^{\mathrm{b}}$} & \multicolumn{1}{c}{$\omega 3^{\mathrm{b}}$} \\
\hline$r \leq r_{\alpha}$ & 4527 & $138(3.0 \pm 0.26 \%)$ & 4598 & $153(3.0 \pm 0.25 \%)$ \\
$r_{\alpha}<r \leq r_{\beta}$ & $2469^{\mathrm{c}}+1840^{\mathrm{d}}$ & $67^{\mathrm{c}}+103^{\mathrm{d}}(4.0 \pm 0.31 \%)$ & $2504^{\mathrm{c}}+1913^{\mathrm{d}}$ & $77^{\mathrm{c}}+67^{\mathrm{d}}(3.0 \pm 0.25 \%)$ \\
$r>r_{\beta}$ & 3492 & $286(8.0 \pm 0.5 \%)$ & 3209 & $195(6.0 \pm 0.44 \%)$ \\
Total & 12328 & $594(5.0 \pm 0.21 \%)$ & 12224 & $492(4.0 \pm 0.18 \%)$ \\
\hline
\end{tabular}

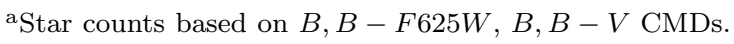

${ }^{\mathrm{b}} \mathrm{Star}$ counts based on $B, B-F 658 N, B, U-V$ CMDs.

${ }^{\mathrm{c}}$ Star counts based on ACS data $\left(r_{\alpha}<r \leq r_{a c s}\right)$.

${ }^{\mathrm{d}}$ Star counts based on WFI data $\left(r_{a c s}<r \leq r_{\beta}\right)$. 
Table 4. Empirical star counts in different "windows" along the RGB. Star counts have been performed in different CMDs.

\begin{tabular}{ccccccc}
\hline \hline$\Delta m_{B}$ & $r \leq r_{\alpha}{ }^{\mathrm{a}}$ & $r_{\alpha}<r \leq r_{\beta}{ }^{\mathrm{a}}$ & $r>r_{\beta}{ }^{\mathrm{a}}$ & $r \leq r_{\alpha}{ }^{\mathrm{b}}$ & $r_{\alpha}<r \leq r_{\beta}{ }^{\mathrm{b}}$ & $r>r_{\beta}{ }^{\mathrm{b}}$ \\
\hline $17.6-16.6$ & 2413 & $2221^{\mathrm{c}}$ & 1837 & 2450 & $2277^{\mathrm{c}}$ & 1668 \\
$17.5-16.5$ & 2187 & 2060 & 1705 & 2228 & 2111 & 1555 \\
$17.4-16.4$ & 2016 & 1890 & 1544 & 2061 & 1953 & 1408 \\
$17.3-16.3$ & 1842 & 1750 & 1411 & 1885 & 1793 & 1294 \\
$17.2-16.2$ & 1639 & 1584 & 1285 & 1685 & 1627 & 1177 \\
$17.1-16.1$ & 1480 & 1448 & 1184 & 1515 & 1489 & 1081 \\
$17.0-16.0$ & 1369 & 1340 & 1072 & 1405 & 1375 & 988 \\
$16.9-15.9$ & 1241 & 1246 & 983 & 1269 & 1278 & 903 \\
$16.8-15.8$ & 1138 & 1168 & 903 & 1164 & 1202 & 835 \\
$16.7-15.7$ & 1084 & 1133 & 828 & 1103 & 1169 & 774 \\
$16.6-5.6$ & 1010 & 1058 & 787 & 1030 & 1083 & 738 \\
\hline
\end{tabular}

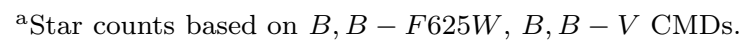

bStar counts based on $B, B-F 658 N, B, U-V$ CMDs.

${ }^{\mathrm{c}}$ Star counts account for both ACS and WFI data. 
Table 5. Theoretical predictions concerning the different lifetimes that a stellar structure with canonical He abundance $(Y \sim 0.23)$, but different stellar masses and chemical compositions spent in the different "windows" along the RGB. The numbers in parentheses are power of ten.

\begin{tabular}{cccccc}
\hline \hline $\begin{array}{c}\Delta m_{B} \\
(\mathrm{mag})\end{array}$ & $\begin{array}{c}\Delta M_{B}^{\mathrm{a}} \\
(\mathrm{mag})\end{array}$ & $\begin{array}{c}\Delta t^{\mathrm{b}} \\
(\mathrm{Z}=0.0002)\end{array}$ & $\begin{array}{c}\Delta t^{\mathrm{b}} \\
(\mathrm{Z}=0.001)\end{array}$ & $\begin{array}{c}\Delta t^{\mathrm{c}} \\
(\mathrm{Z}=0.0002)\end{array}$ & $\begin{array}{c}\Delta t^{\mathrm{c}} \\
(\mathrm{Z}=0.001)\end{array}$ \\
\hline $17.60-16.61$ & $3.44-2.44$ & $2.33(8)$ & $2.08(8)$ & $2.45(08)$ & $2.01(8)$ \\
$17.50-16.51$ & $3.34-2.35$ & $2.13(8)$ & $1.90(8)$ & $2.05(08)$ & $1.86(8)$ \\
$17.40-16.40$ & $3.24-2.24$ & $1.97(8)$ & $1.76(8)$ & $1.82(08)$ & $1.74(8)$ \\
$17.30-16.30$ & $3.14-2.14$ & $1.81(8)$ & $1.62(8)$ & $1.70(08)$ & $1.60(8)$ \\
$17.20-16.20$ & $3.04-2.04$ & $1.68(8)$ & $1.49(8)$ & $1.58(08)$ & $1.47(8)$ \\
$17.10-16.11$ & $2.94-1.95$ & $1.55(8)$ & $1.37(8)$ & $1.49(08)$ & $1.36(8)$ \\
$17.00-16.00$ & $2.84-1.84$ & $1.43(8)$ & $1.27(8)$ & $1.39(08)$ & $1.23(8)$ \\
$16.90-15.91$ & $2.74-1.75$ & $1.31(8)$ & $1.16(8)$ & $1.29(08)$ & $1.14(8)$ \\
$16.80-15.80$ & $2.64-1.64$ & $1.21(8)$ & $1.05(8)$ & $1.18(08)$ & $1.06(8)$ \\
$16.70-15.70$ & $2.54-1.54$ & $1.11(8)$ & $9.75(7)$ & $1.08(08)$ & $9.76(7)$ \\
$16.60-15.62$ & $2.44-1.45$ & $1.01(8)$ & $8.96(7)$ & $1.00(08)$ & $8.91(7)$ \\
\hline
\end{tabular}

${ }^{a}$ Absolute $B$-band magnitudes for stellar structures with $M=0.80 M_{\odot}, \mathrm{Z}=0.0002$, and $\mathrm{Y}=0.23$.

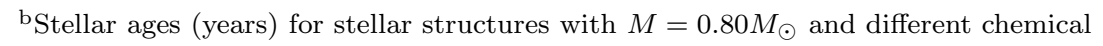
compositions.

${ }^{\mathrm{c}}$ Stellar ages (years) for a stellar structure with $M=0.85 M_{\odot}$ and different chemical compositions. 
Table 6. Theoretical predictions concerning the different lifetimes that a stellar structure at fixed mass value but with different metal abundances and a He-enhanced ( $Y=0.33,0.42)$ composition spend in the different "windows" along the RGB. The numbers in parentheses are power of ten.

\begin{tabular}{cccccc}
\hline \hline $\begin{array}{c}\Delta m_{B} \\
(\mathrm{mag})\end{array}$ & $\begin{array}{c}\Delta M_{B}{ }^{\mathrm{a}} \\
(\mathrm{mag})\end{array}$ & $\begin{array}{c}\Delta t^{\mathrm{b}} \\
(\mathrm{Z}=0.0002)\end{array}$ & $\begin{array}{c}\Delta t^{\mathrm{b}} \\
(\mathrm{Z}=0.001)\end{array}$ & $\begin{array}{c}\Delta t^{\mathrm{c}} \\
(\mathrm{Z}=0.0002)\end{array}$ & $\begin{array}{c}\Delta t^{\mathrm{c}} \\
(\mathrm{Z}=0.001)\end{array}$ \\
\hline $17.61-16.60$ & $2.90-1.84$ & $2.17(8)$ & $1.78(8)$ & $2.16(8)$ & $1.77(8)$ \\
$17.50-16.50$ & $2.78-1.73$ & $1.97(8)$ & $1.66(8)$ & $1.91(8)$ & $1.62(8)$ \\
$17.41-16.41$ & $2.69-1.63$ & $1.84(8)$ & $1.53(8)$ & $1.78(8)$ & $1.50(8)$ \\
$17.31-16.31$ & $2.58-1.52$ & $1.70(8)$ & $1.41(8)$ & $1.64(8)$ & $1.39(8)$ \\
$17.21-16.21$ & $2.48-1.42$ & $1.58(8)$ & $1.31(8)$ & $1.48(8)$ & $1.28(8)$ \\
$17.11-16.11$ & $2.37-1.31$ & $1.45(8)$ & $1.20(8)$ & $1.35(8)$ & $1.18(8)$ \\
$17.01-16.00$ & $2.27-1.20$ & $1.35(8)$ & $1.10(8)$ & $1.25(8)$ & $1.08(8)$ \\
$16.91-15.90$ & $2.16-1.10$ & $1.23(8)$ & $1.01(8)$ & $1.15(8)$ & $9.78(7)$ \\
$16.81-15.80$ & $2.06-0.99$ & $1.14(8)$ & $9.24(7)$ & $1.05(8)$ & $8.91(7)$ \\
$16.70-15.70$ & $1.94-0.88$ & $1.03(8)$ & $8.45(7)$ & $9.64(7)$ & $8.22(7)$ \\
$16.60-15.60$ & $1.84-0.77$ & $9.47(7)$ & $7.75(7)$ & $8.72(7)$ & $7.60(7)$ \\
\hline
\end{tabular}

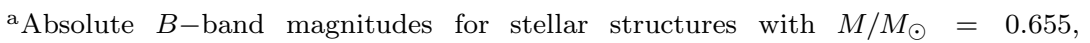
$\mathrm{Z}=0.0002$, and $\mathrm{Y}=0.33$.

${ }^{\mathrm{b}}$ Stellar ages (years) for helium enhanced $(\mathrm{Y}=0.33)$ stellar structures constructed at fixed TO-age $(12 \mathrm{Gyr})$ and metal abundance. The TO-mass are $M / M_{\odot}=0.655$, for $\mathrm{Z}=0.0002$ and $M / M_{\odot}=0.665$ for $\mathrm{Z}=0.001$.

${ }^{\mathrm{c}}$ Stellar ages (years) for helium enhanced $(\mathrm{Y}=0.42)$ stellar structures constructed at fixed TO-age (12 Gyr) and metal abundance. The TO-mass is $M / M_{\odot}=0.55$, for both $\mathrm{Z}=0.0002$ and and $\mathrm{Z}=0.001$ structures. 


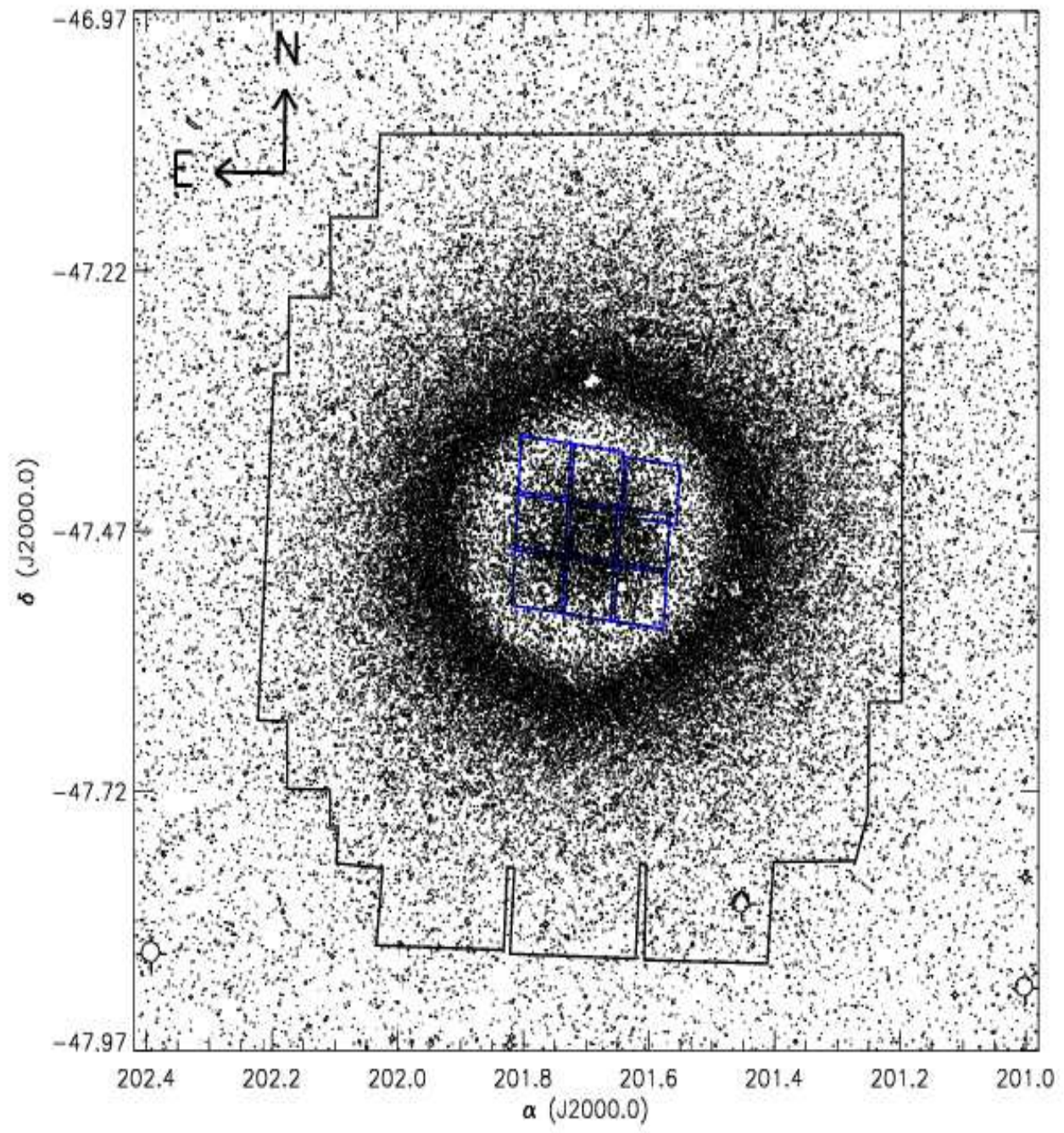

Fig. 1.- Location of the pointings of the ground-based $U, B, V, I$-band data collected with WFI@2.2m ESO/MPI (black line) and the space $F 435 W, F 625 W, F 658 N$-band data collected with ACS@HST (blue mosaic). The outline of individual WFI pointings is plotted over a $1 \times 1$ square degree field in the Digital Sky Survey (DSS). The central cluster field, unresolved by the DSS, is superposed by a sample from our star catalogue to show the location of the HST mosaic. 


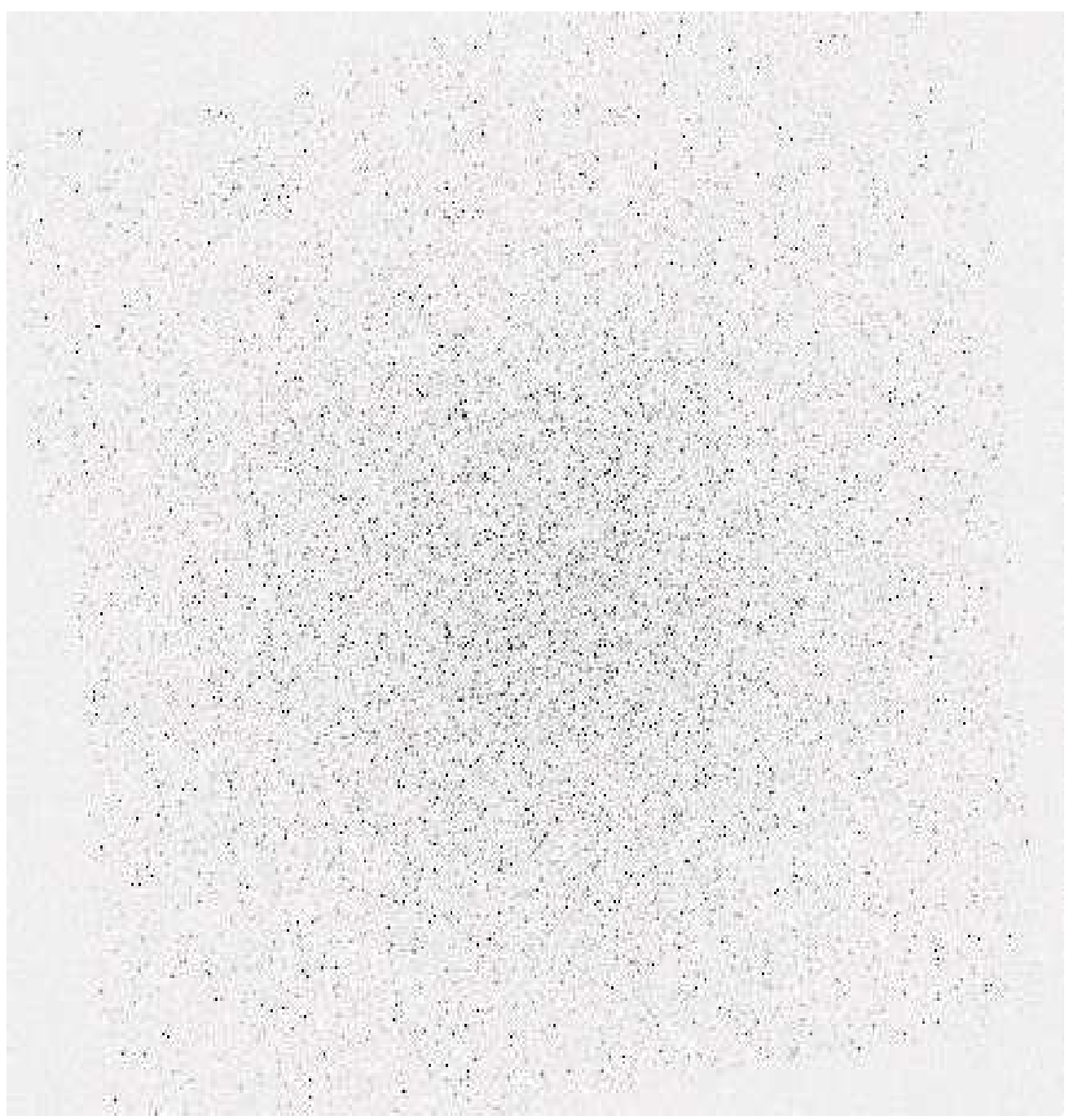

Fig. 2.- The color image is a true color hybrid composition of WFI and ACS images. The catalogue based on ACS images provides the spatial information, while the colors of individual stars are based on $U, B, V, I$-band WFI data. In order to preserve the appropriate resolution three artificial images of $8192 \times 8192$ pixels have been created: one for the combined $U$ and $B$-band data, and two for the $V$ and the $I$-band data. Approximately 1 million stars have been included in each of these images using the artdata.mkobjects task, resulting in an image with almost the same pixel scale as an ACS image. The three independent channels have then been merged to create a true-color image. The mosaic's field on the sky is $\approx 10^{\prime} \times 9.5^{\prime}$. north is up, and east is to the left. 


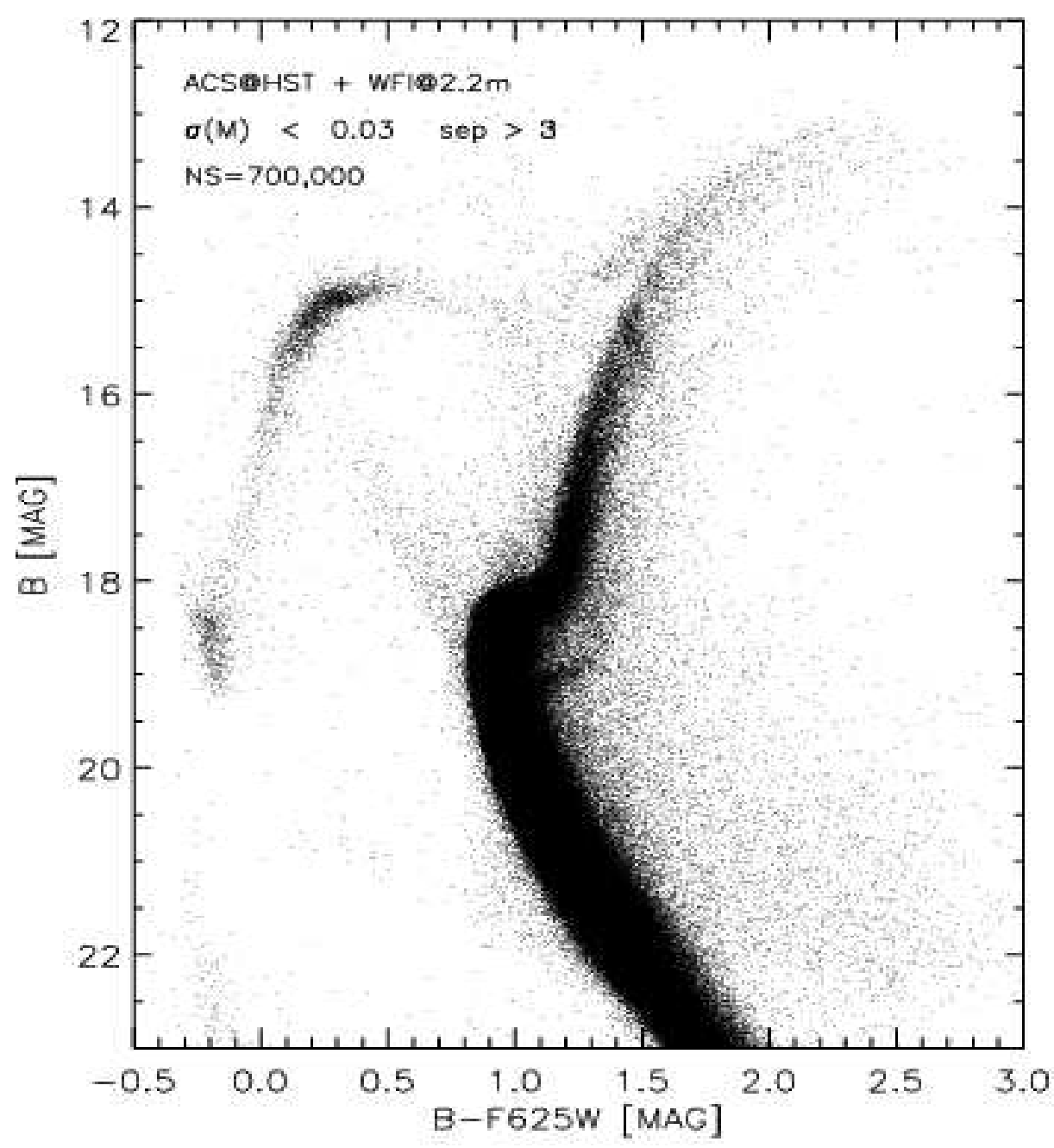

Fig. 3.- Composite $B, B-F 625 W$ diagram of the entire field. ACS data in the $F 435 W$-band were transformed into the $B$-band, while $V$ and $I$ WFI data have been transformed into $\mathrm{F} 625 \mathrm{~W}$ using the transformation $F 625 W=V \times 0.544+I \times 0.455 \mathrm{mag}$. See text for more details. 

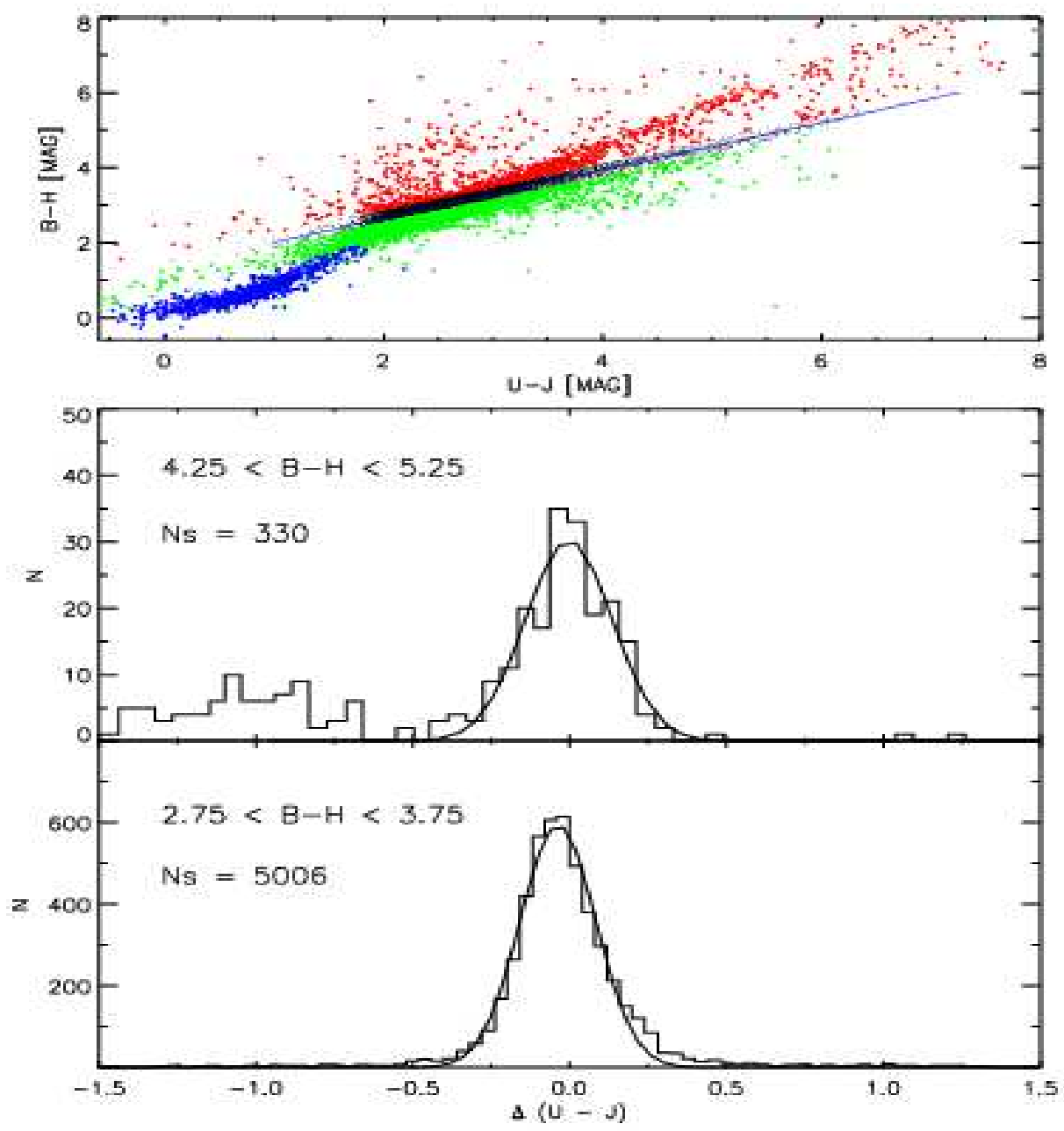

Fig. 4.- Top: Optical-NIR color-color plane for bright stars in $\omega$ Cen. Black and blue dots display likely cluster RG and HB stars, while red and green dots likely field objects. The solid blue line shows the fitting line adopted to select likely field and cluster stars. Middle: distance in $U-J$ color from the fitting line for the stars with $4.25 \leq B-H \leq 5.25$ and located at a radial distance ranging from 2.5 to 10 arcmin from the cluster center. The solid line shows the gaussian that fits the color distribution. Bottom: Same as the middle, but for stars with $2.75 \leq B-H \leq 3.75$. 


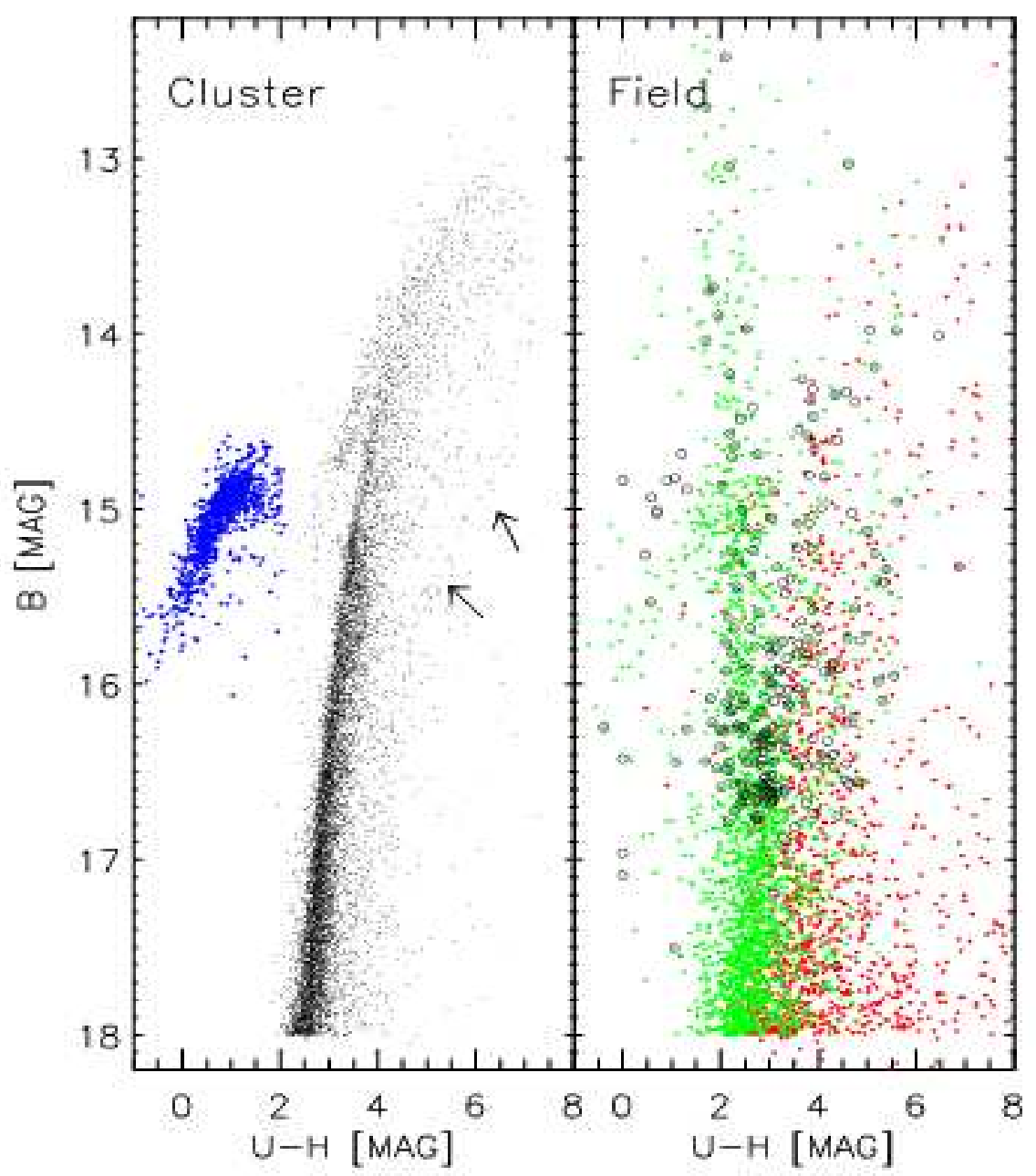

Fig. 5.- Left: Optical-NIR CMD for bright likely cluster stars in $\omega$ Cen. Different samples have the same colors as in Fig. 3. Right: same as left, but for likely field objects. Open circles mark objects that according to proper motion measurements (van Leeuwen et al. $2000)$ present a low membership probability $(P \leq 10 \%)$. 


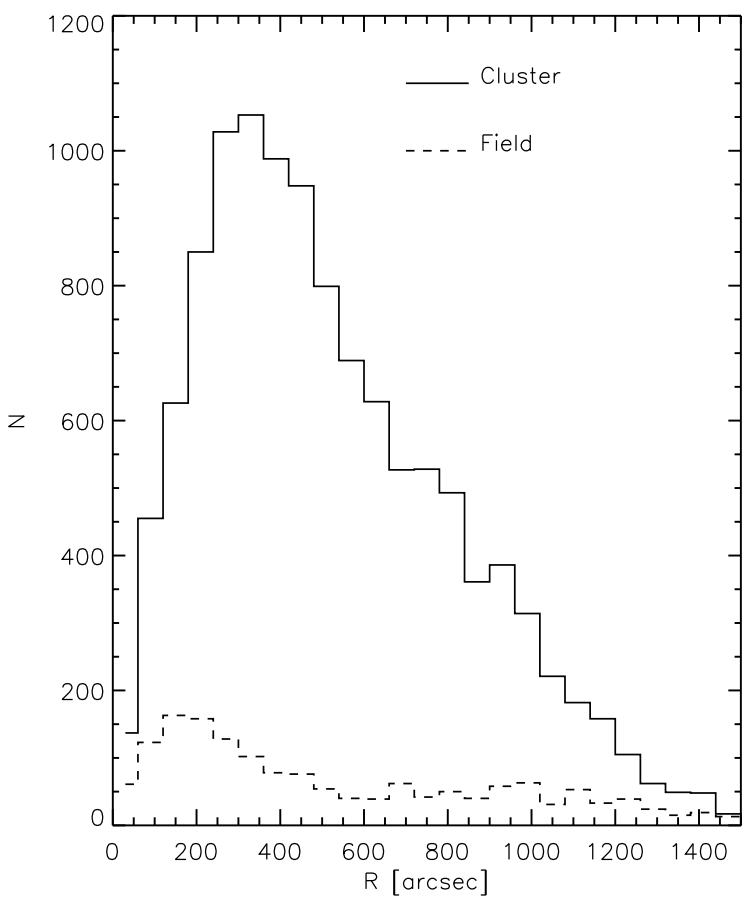

Fig. 6.- Radial distribution of likely field objects (dashed line) and cluster (solid line) stars. 


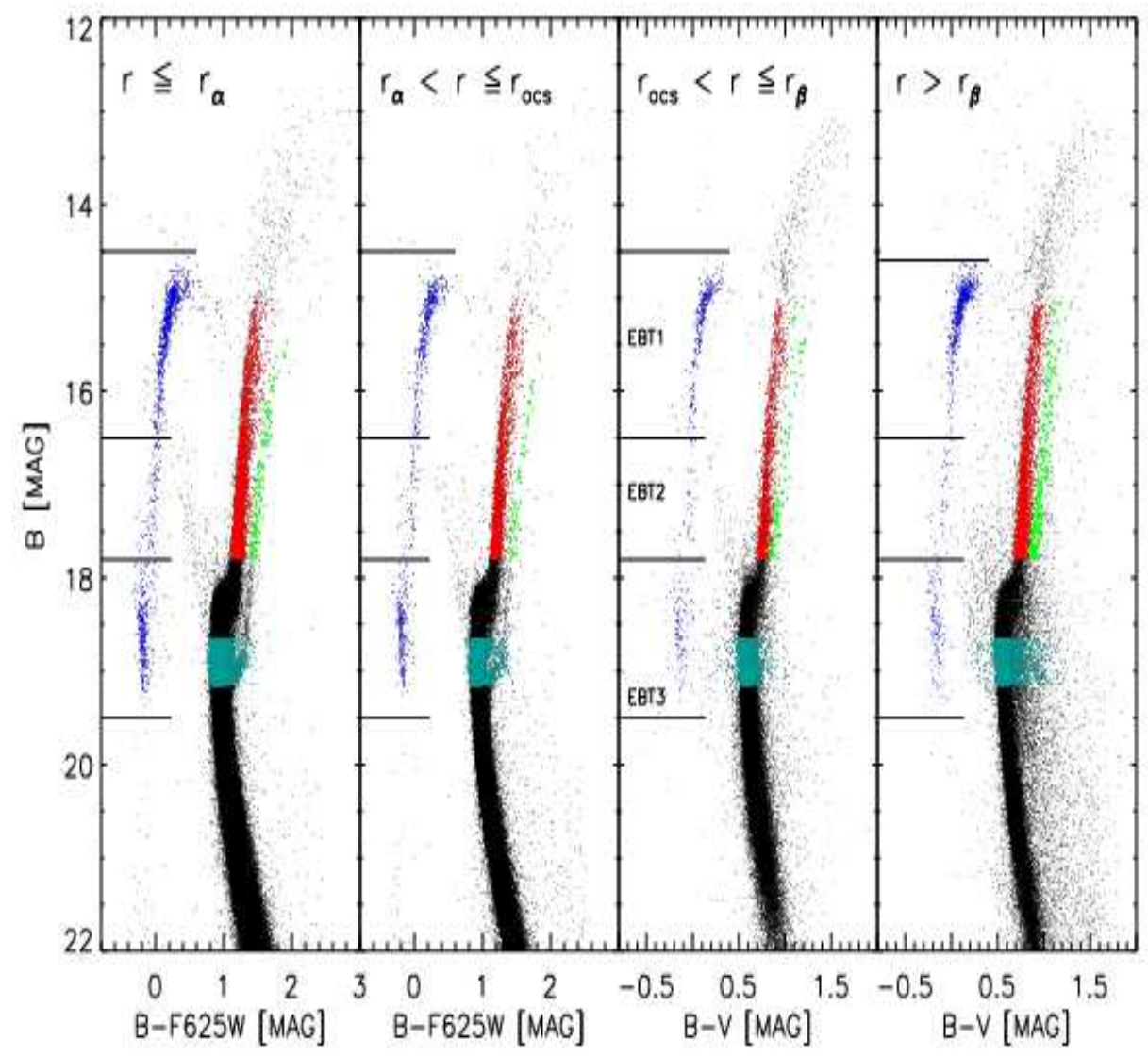

Fig. 7.- CMDs as a function of the radial distance based on ACS@HST $(B, B-F 625 W)$ and ground-based $(B, B-V)$ data. The bulk of the stars plotted in the different CMDs (black dots) were selected using different criteria (sep, photometric errors, sharpness). Blue dots mark HB stars, red dots RGB stars, green dots stars belonging to the $\omega 3$-branch, and cyan dots MS stars adopted for the star counts. These sub-samples have only been selected between upper and lower magnitude limits and cleaned for field star contamination. The solid lines mark from top to bottom the EBT1, the EBT2, and the EBT3 region. 


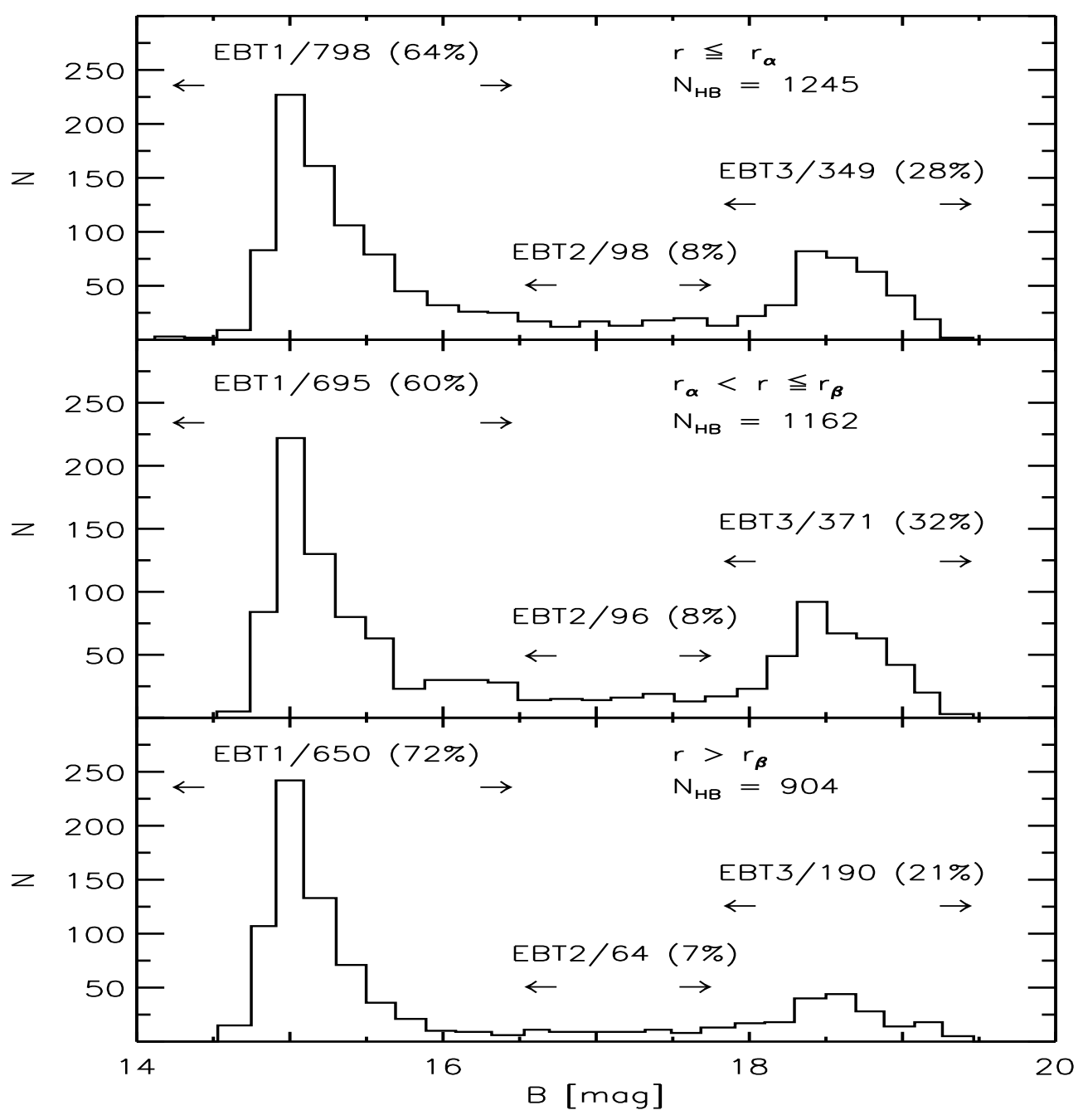

Fig. 8. - The $B$-band luminosity function of HB stars from the very center (top) to more external cluster regions (middle, bottom). The arrows mark the magnitude interval of the three selected EBT regions (see solid lines in Fig. 7). The total number of HB stars, the star counts for each sub-sample, and their relative fractions in each region are also labeled. 


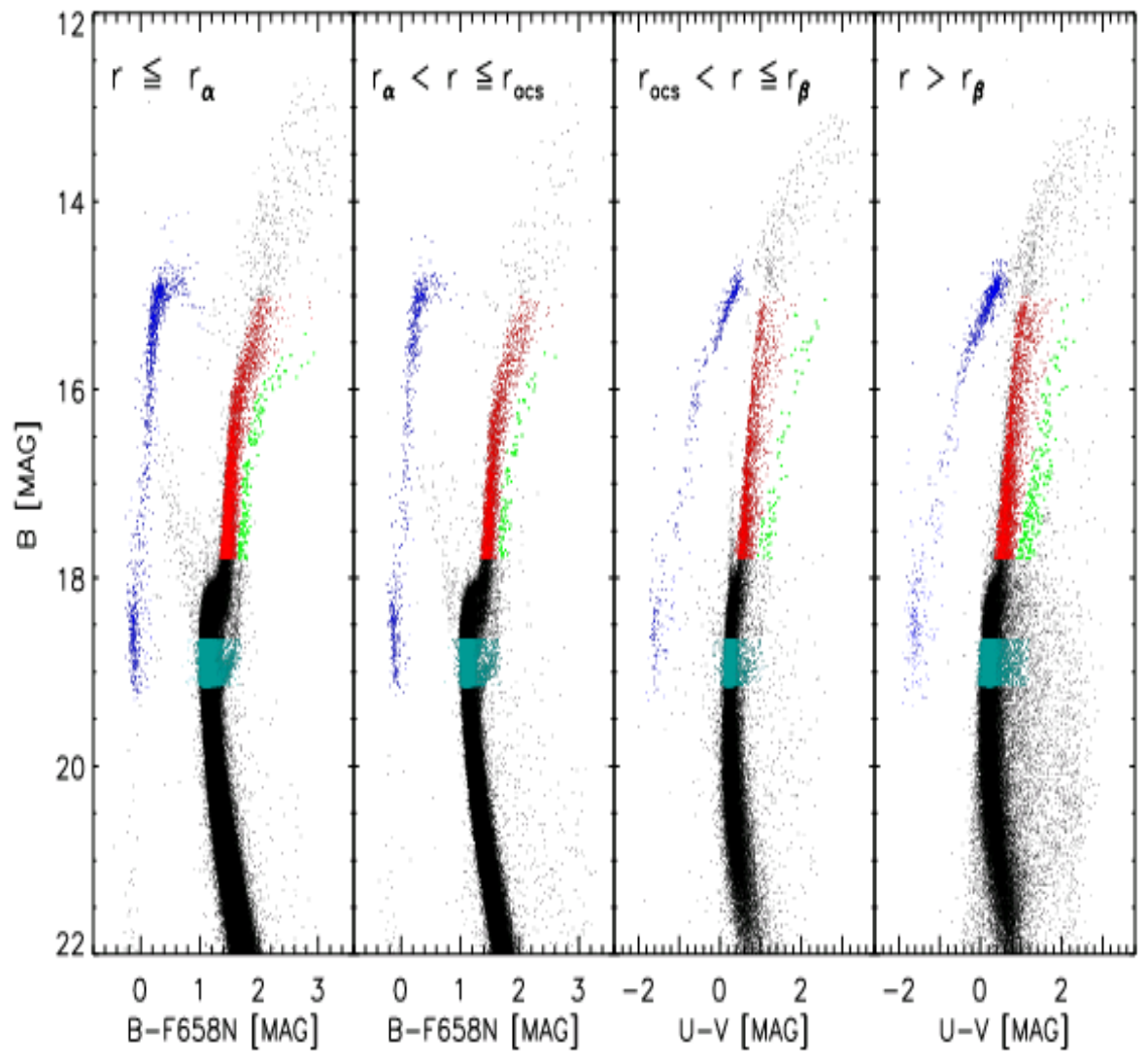

Fig. 9.- Same as Fig. 7, but based on ACS@HST ( $B, B-F 658 N)$ and ground-based WFI@2.2m ESO/MPI $(B, U-V)$ data. 


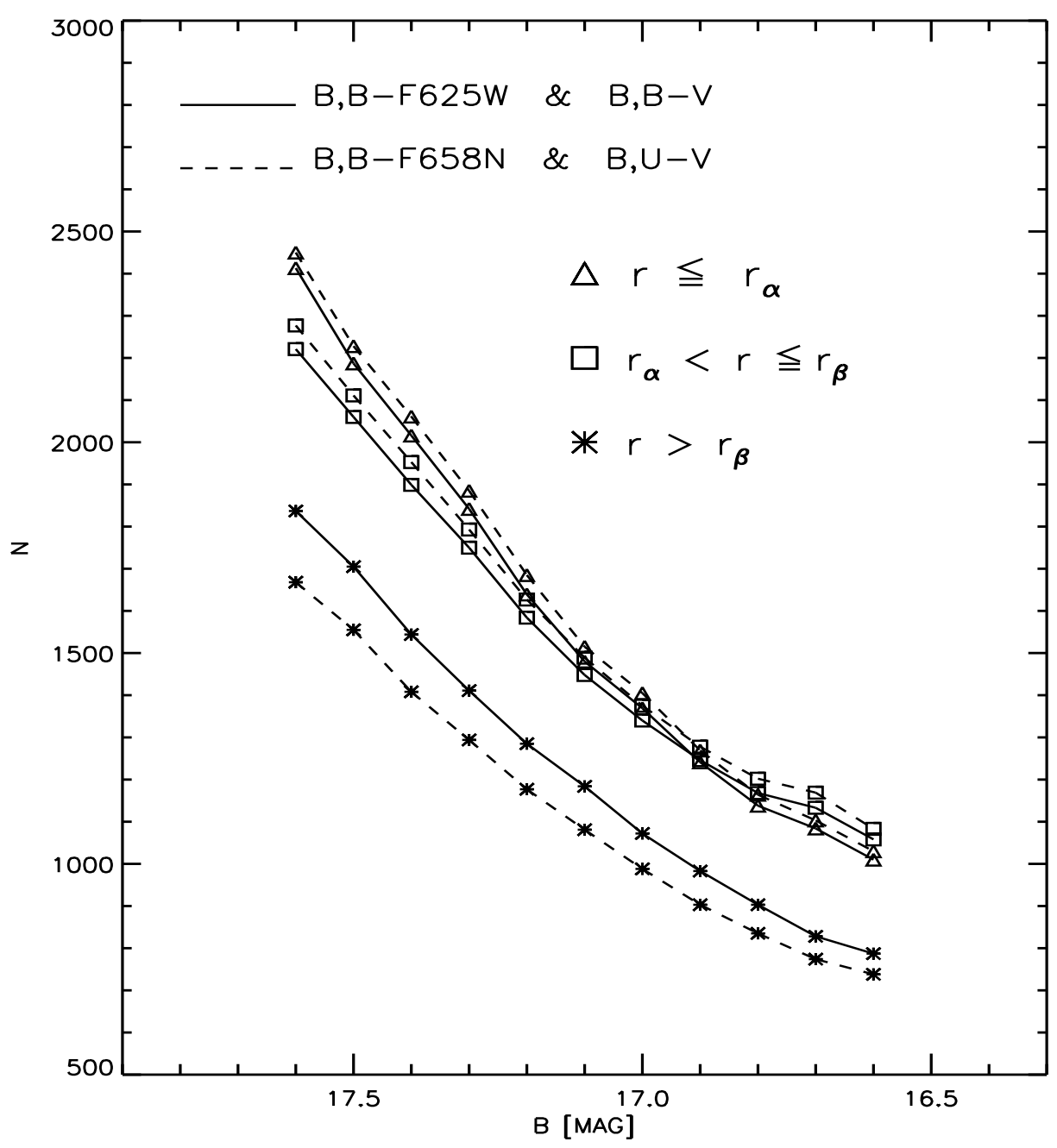

Fig. 10.- Empirical star counts along the RGB of $\omega$ Cen. Individual bins cover an interval of one $B$-magnitude and have been plotted at the faint end of the magnitude bin. Different symbols mark star counts in different annuli. Solid and dashed lines show RG samples selected in different CMDs. 


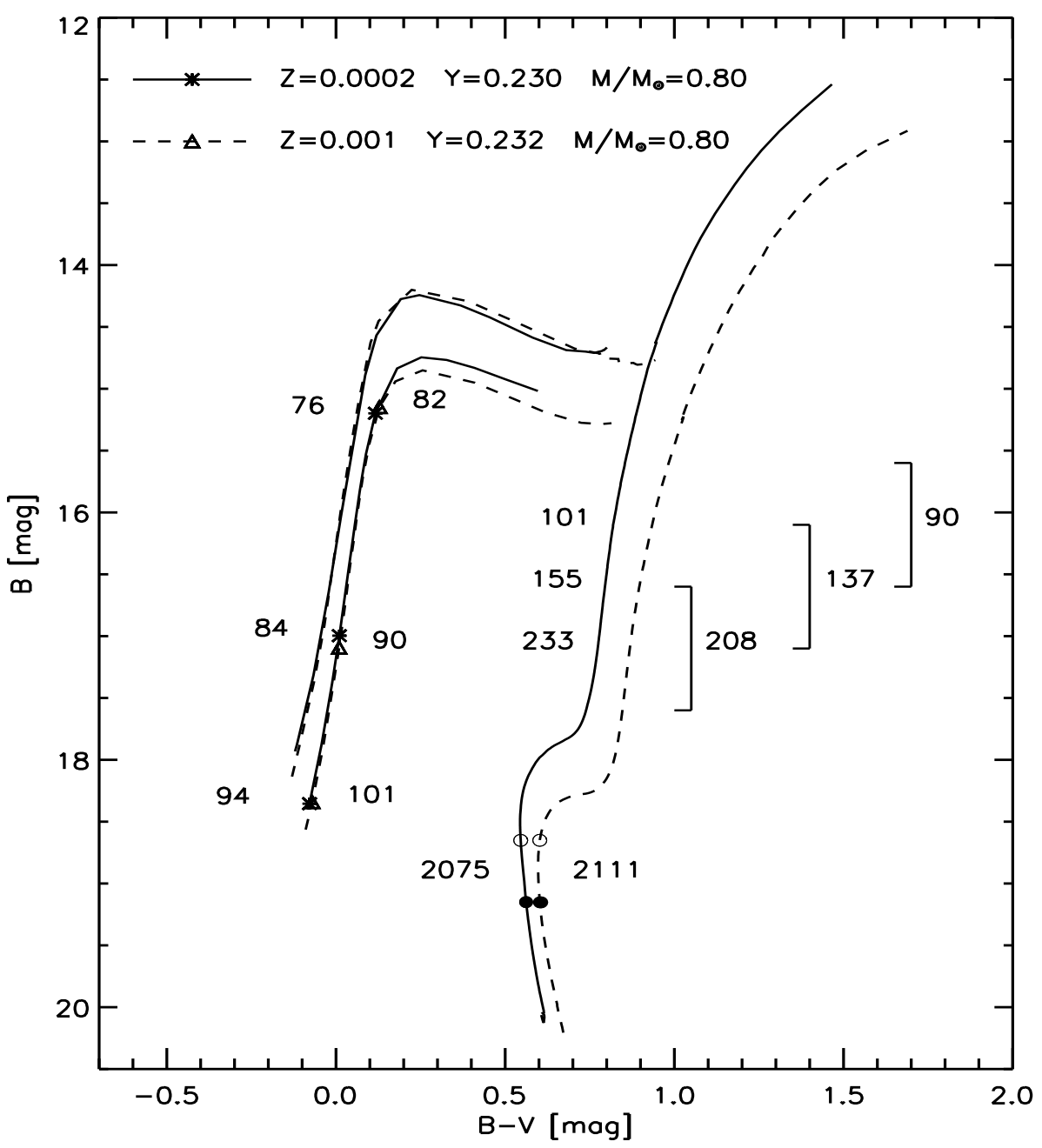

Fig. 11.- Evolutionary predictions in the $B, B-V$ CMD at fixed mass $\left(M=0.80 M_{\odot}\right)$ and different chemical compositions (see labeled values). The square brackets along the evolutionary tracks mark three out of the eleven one magnitude bins along the RGB. The labels display the predicted lifetimes (Myr) for both metal-poor (left, $Z=0.0002$ ) and more metal-rich (right, $\mathrm{Z}=0.001$ ) structures. Solid and dashed lines for He burning structures show the ZAHB and the exhaustion of central He burning with different chemical compositions. The labels and the symbols along the ZAHBs display predicted lifetimes (Myr) of metal-poor (left, asterisks) and more metal-rich (right, triangles) He burning structures representative from top to bottom of EBT1, EBT2, and EBT3 groups. Solid and empty circles mark the region of the MS adopted to estimate the MS lifetime. 


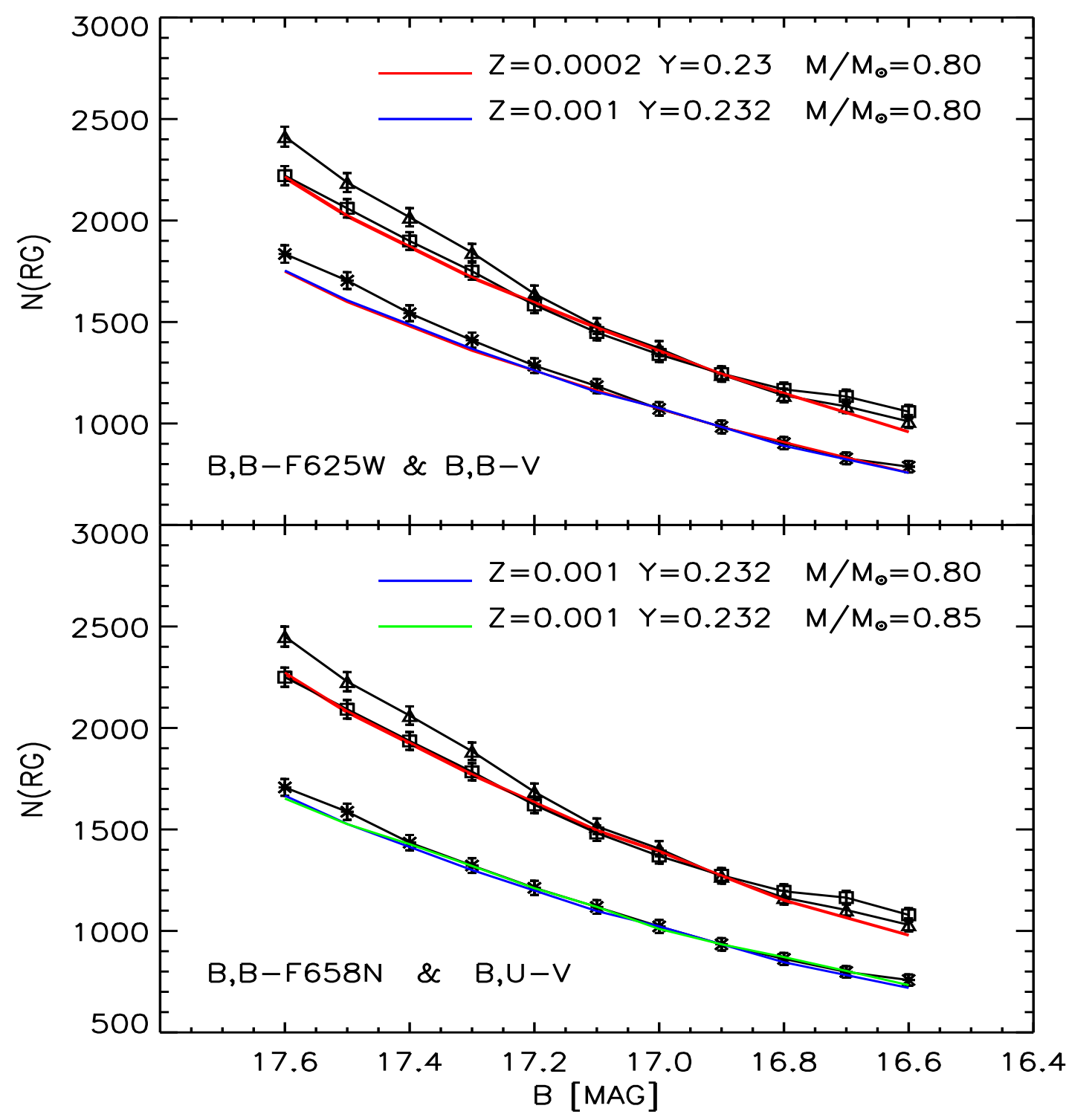

Fig. 12.- Top: Comparison between empirical star counts along the RGB of $\omega$ Cen and predicted lifetimes for different chemical compositions and fixed stellar mass (see labeled values). Theoretical predictions have been normalized to observations in the magnitude bin $m_{B}=16.9-15.9\left(M_{B}=2.74-1.75\right)$. The normalization was separately applied to the star counts of the three different annuli. The symbols are the same as in Fig. 10 and the error bars only account for uncertainties (Poisson) on star counts. Bottom: Same as the top, but for RG stars selected in the CMDs $B, B-F 652 N$ (ACS); $B, U-V$ (WFI). Predicted lifetimes refer to stellar structures constructed at fixed chemical composition and different stellar masses (see labeled values). 


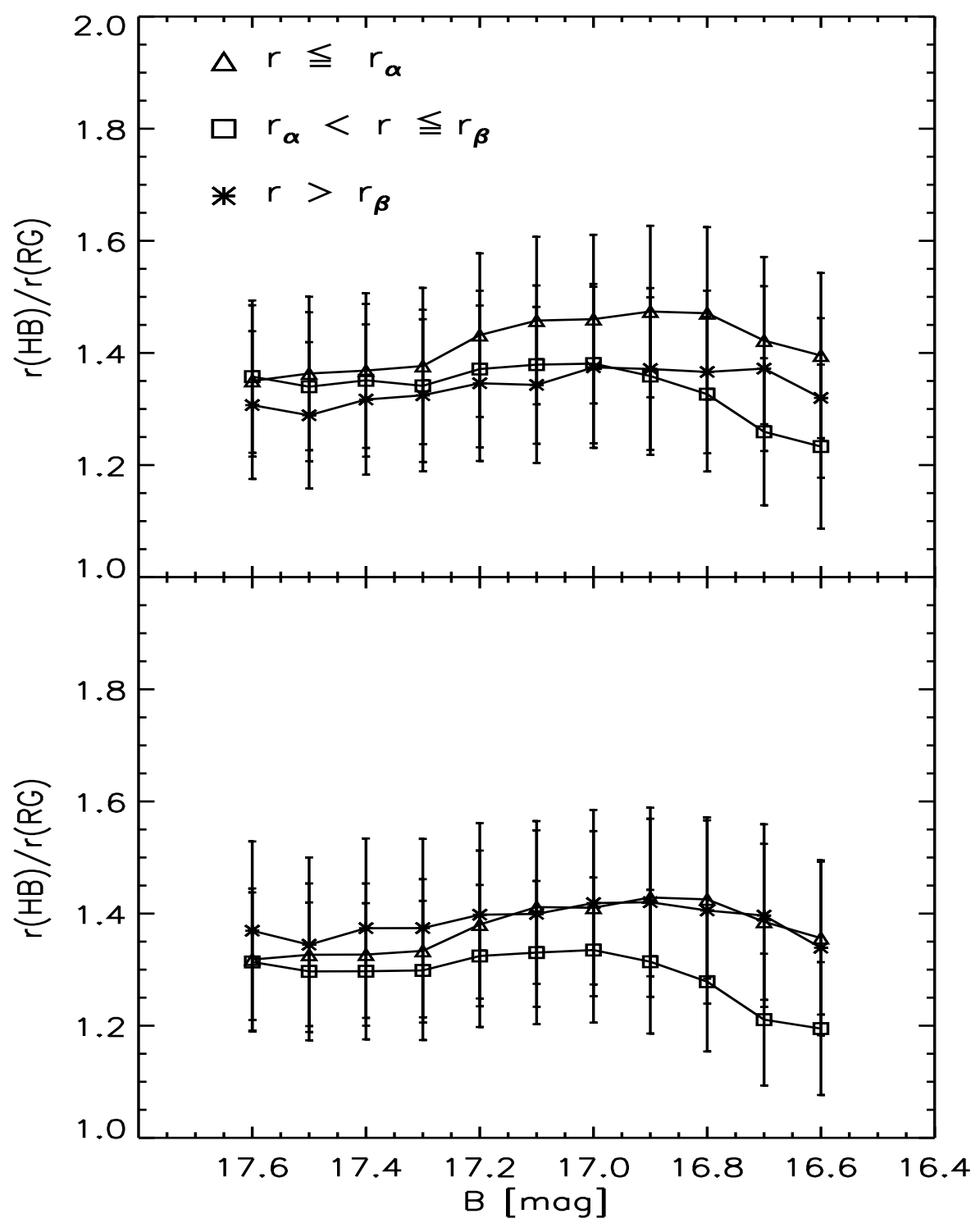

Fig. 13.- Top: Ratios between the rate of $\mathrm{HB}$ stars and the rate of RG stars in the three different annuli. Star counts are based on $B, B-F 625 W$ (ACS) and $B, B-V$ (WFI) CMDs. The symbols are the same as in Fig. 10 and the error bars account for uncertainties on star counts (Poisson) and on evolutionary lifetimes (10\%). Bottom: Same as the top, but for star counts based on $B, B-F 658 N$ (ACS) and $B, U-V$ (WFI) CMDs. 


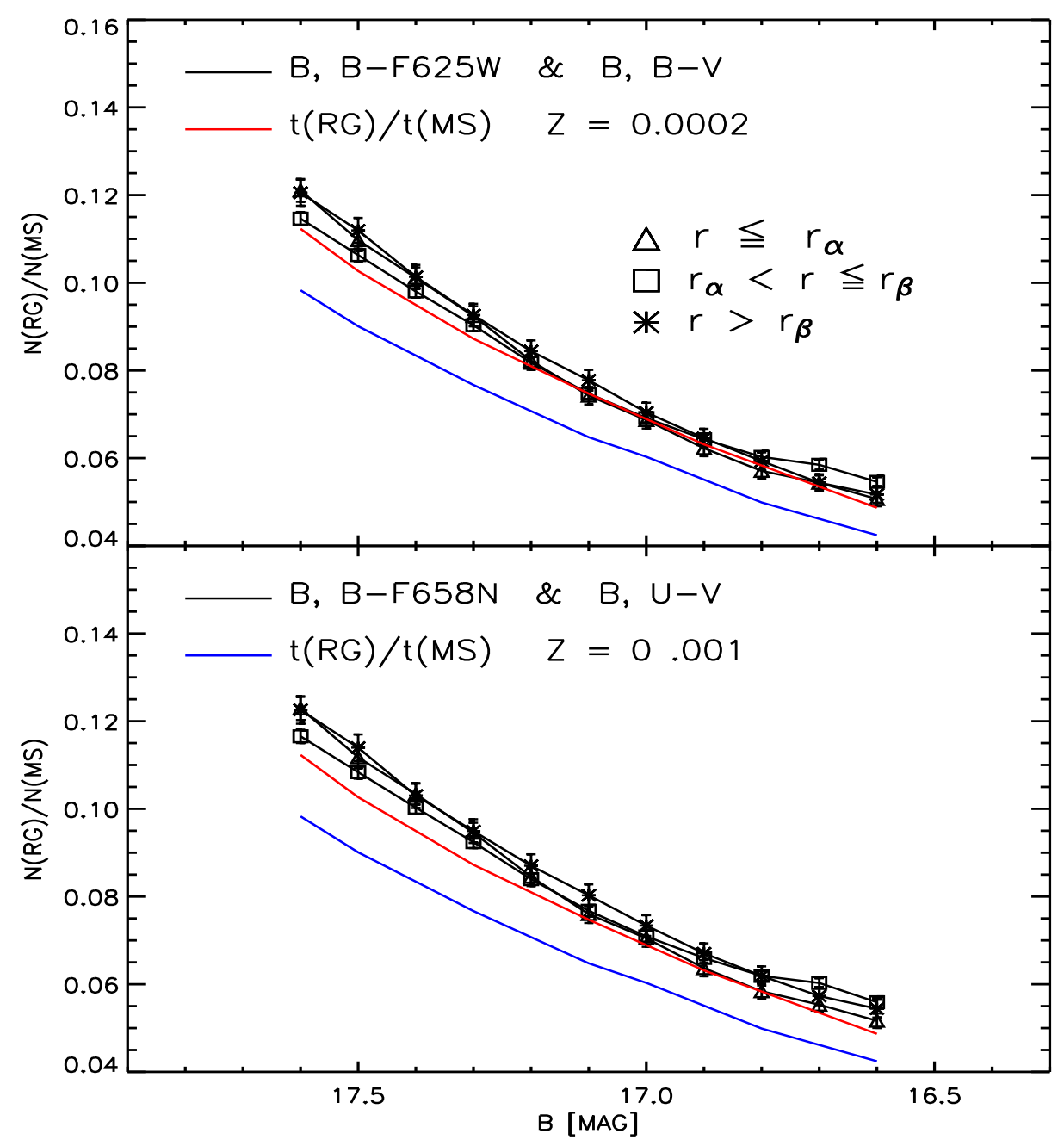

Fig. 14.- Top: Ratio between RG and MS star counts selected in the $B, B-F 625 W$ (ACS) and $B, B-V$ (WFI) CMDs. The red and the blue lines show the predicted ratio between RG and MS lifetimes at fixed stellar mass $\left(M=0.80 M_{\odot}\right)$ and different metal abundances (see labeled values). Theoretical predictions were not normalized to observed ratios. The symbols are the same as in Fig. 10 and the error bars only account for uncertainties (Poisson) in star counts. Bottom: Same as the top, but the RG and MS star counts were selected in the $B, B-F 658 N(\mathrm{ACS})$ and $B, U-V$ (WFI) CMDs. 


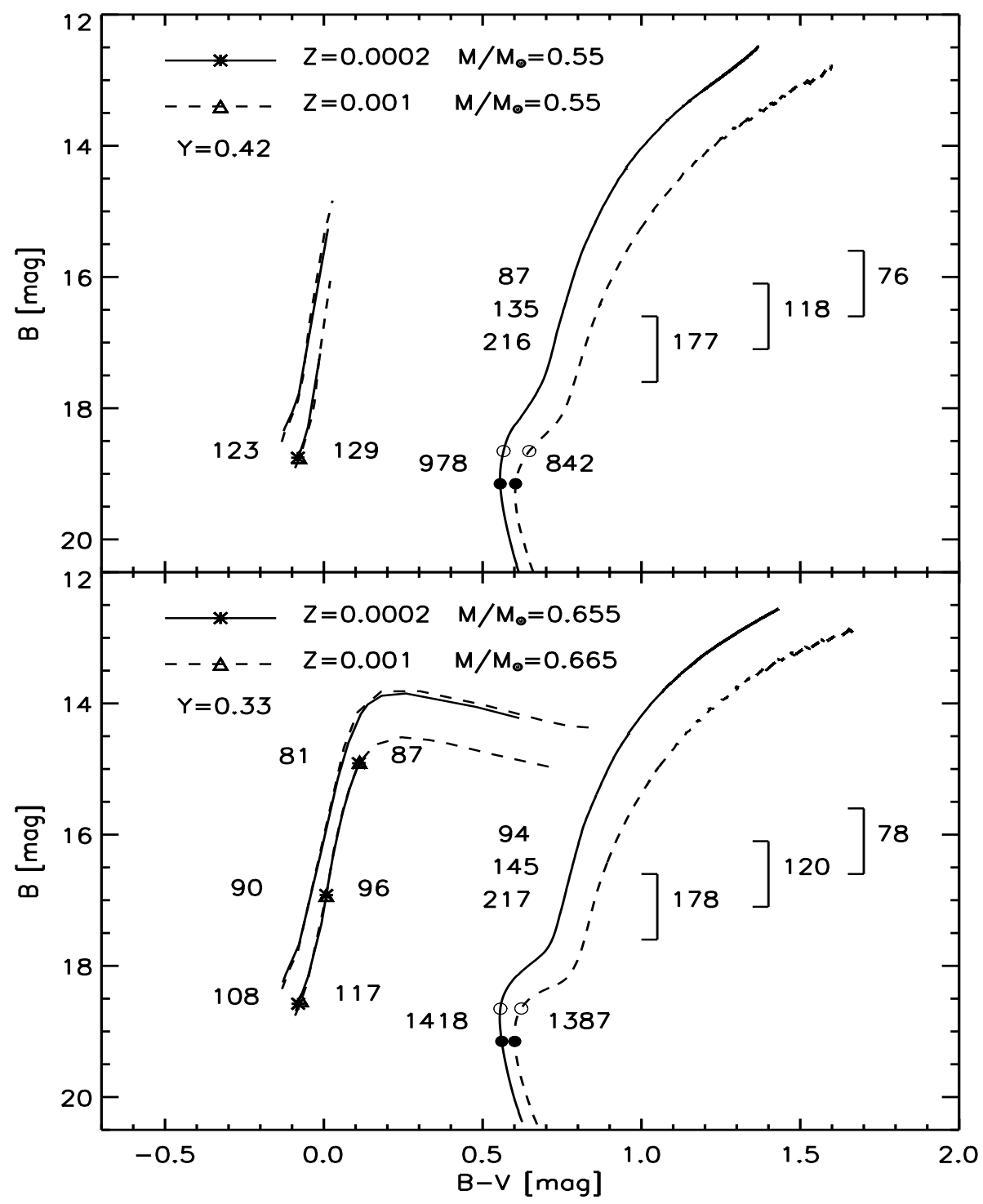

Fig. 15.- Top: Same as Fig. 11. The evolutionary models refer to stellar structures constructed by adopting the same TO-age and metal abundances but a higher helium content $(\mathrm{Y}=0.42)$. Bottom: Same as the top, but for a lower helium content $(\mathrm{Y}=0.33)$. 


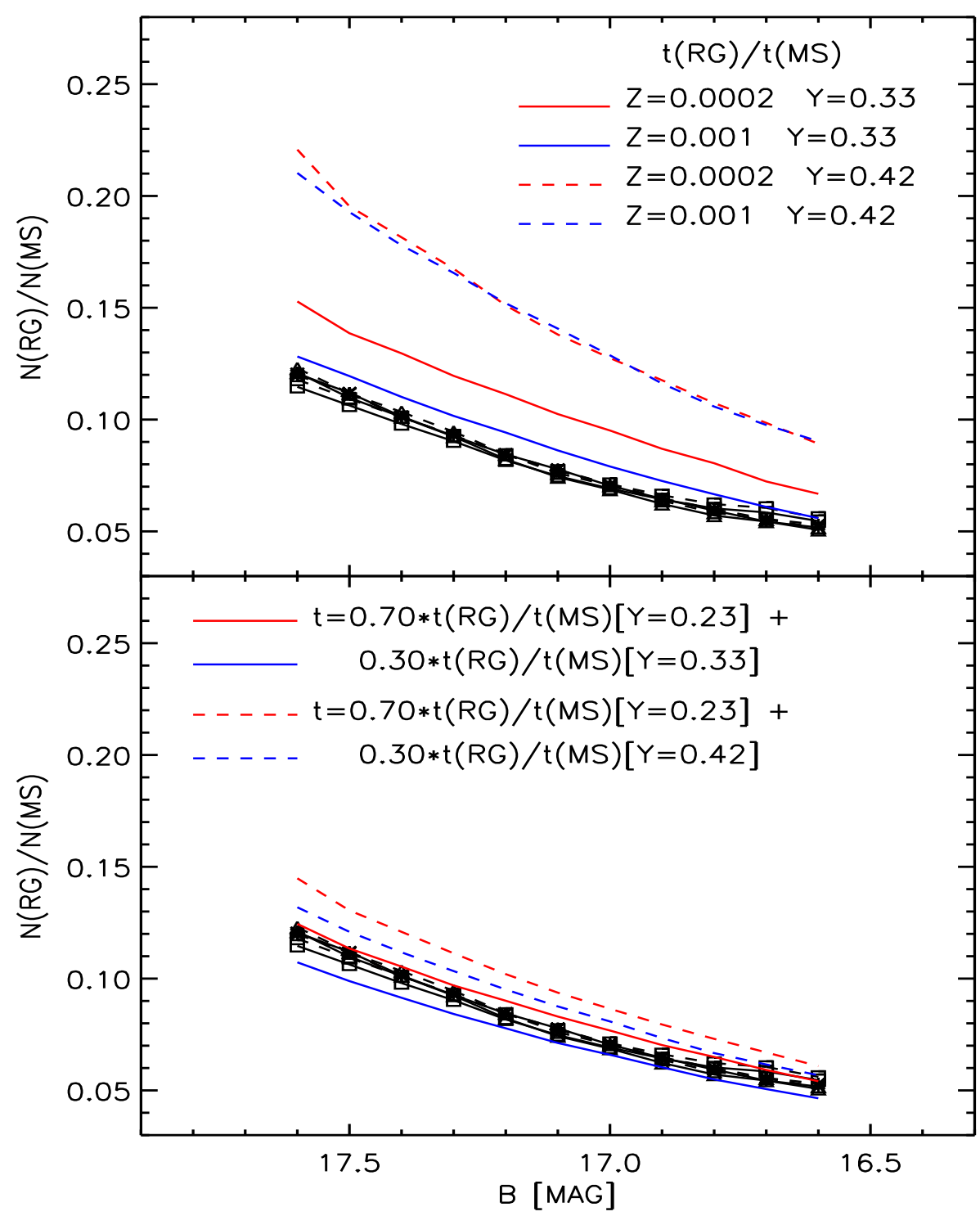

Fig. 16. - Top: Same as Fig. 14. The theoretical ratios refer to the helium enhanced $(Y=0.33,0.42)$ stellar structures. Bottom: Same as the top but predicted ratios account for the fraction of He enhanced stellar population (30\%). See text for more details. 


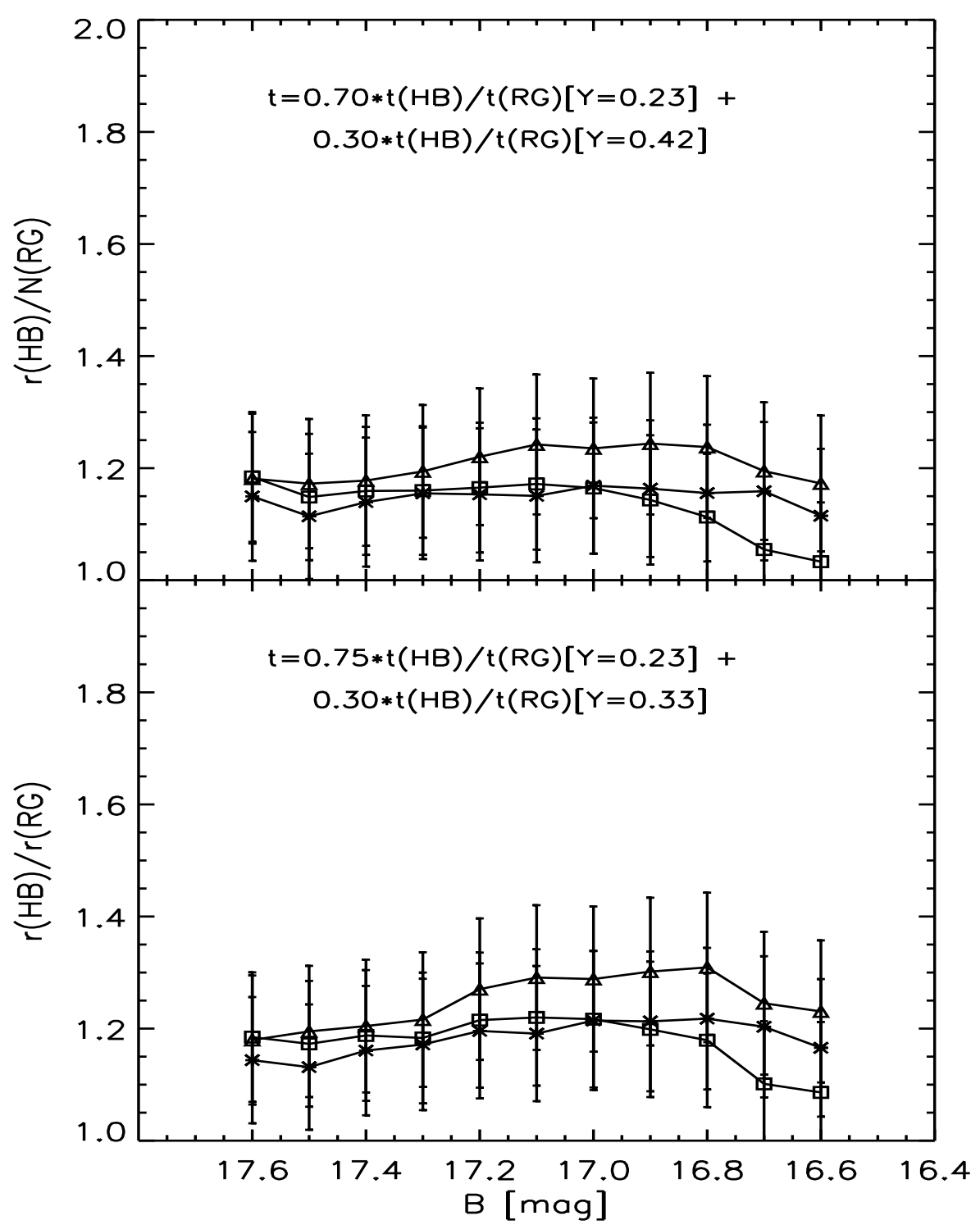

Fig. 17.- Top: Comparison between the rates of HB and RG stars. Star counts are based on $B, B-F 625 W$ (ACS) and $B, B-V$ (WFI) CMDs. The symbols are the same as in Fig. 13 and the error bars account for uncertainties on star counts (Poisson) and on evolutionary lifetimes (10\%). The predicted HB lifetimes account for stellar populations with different fractions of canonical ( $\mathrm{Y}=0.23,70 \%)$ and He-enriched $(\mathrm{Y}=0.42,30 \%)$ stars and for the spread in metal abundance. Bottom: Same as the top, but for a He-enriched stellar population with $\mathrm{Y}=0.33$. 